\title{
New SMOS Sea Surface Salinity with reduced systematic errors and improved variability
}

\author{
Boutin J. ${ }^{1,{ }^{*}}$, Vergely J.L. ${ }^{2}$, Marchand S. ${ }^{1}$, D'Amico F. ${ }^{1}$, Hasson A. ${ }^{1}$, Kolodziejczyk Nicolas ${ }^{4}$, \\ Reul Nicolas ${ }^{3}$, Reverdin G. ${ }^{1}$, Vialard J. ${ }^{1}$
}

\author{
${ }^{1}$ Sorbonne Université, CNRS, IRD, MNHN, Laboratoire d'Océanographie et du Climat: \\ Expérimentations et Approches Numériques (LOCEAN), 75005 Paris, France \\ ${ }^{2}$ ACRI-st, Guyancourt, France \\ ${ }^{3}$ Laboratoire d'Océanographie Physique et Spatiale (LOPS), Univ. Brest, CNRS, Ifremer, IRD, Brest, \\ France \\ *Corresponding author : J. Boutin, email address : jb@locean-ipsl.upmc.fr
}

\begin{abstract}
:
Salinity observing satellites have the potential to monitor river fresh-water plumes mesoscale spatiotemporal variations better than any other observing system. In the case of the Soil Moisture and Ocean Salinity (SMOS) satellite mission, this capacity was hampered due to the contamination of SMOS data processing by strong land-sea emissivity contrasts. Kolodziejczyk et al. (2016) (hereafter K2016) developed a methodology to mitigate SMOS systematic errors in the vicinity of continents, that greatly improved the quality of the SMOS Sea Surface Salinity (SSS). Here, we find that SSS variability, however, often remained underestimated, such as near major river mouths. We revise the K2016 methodology with: a) a less stringent filtering of measurements in regions with high SSS natural variability (inferred from SMOS measurements) and b) a correction for seasonally-varying latitudinal systematic errors. With this new mitigation, SMOS SSS becomes more consistent with the independent SMAP SSS close to land, for instance capturing consistent spatio-temporal variations of low salinity waters in the Bay of Bengal and Gulf of Mexico. The standard deviation of the differences between SMOS and SMAP weekly SSS is $<0.3$ pss in most of the open ocean. The standard deviation of the differences between 18-day SMOS SSS and $100-\mathrm{km}$ averaged ship SSS is 0.20 pss $(0.24$ pss before correction) in the open ocean. Even if this standard deviation of the differences increases closer to land, the larger SSS variability yields a more favorable signal-to-noise ratio, with r2 between SMOS and SMAP SSS larger than 0.8. The correction also reduces systematic biases associated with man-made Radio Frequency Interferences (RFI), although SMOS SSS remains more impacted by RFI than SMAP SSS. This newly-processed dataset will allow the analysis of SSS variability over a larger than 8 years period in regions previously heavily influenced by land-sea contamination, such as the Bay of Bengal or the Gulf of Mexico.
\end{abstract}




\section{Highlights}

- Improved SMOS salinity systematic error correction from Kolodziejczyk et al. (2016) R Refined variability of sea surface salinity near e.g. major river mouths Consistent mesoscale patterns observed by SMOS and SMAP satellite missions

Keywords : SMOS, Sea Surface Salinity, SMAP 


\section{Introduction}

With 8 years and counting, the Soil Moisture and Ocean Salinity (SMOS) European mission (Kerr et al., 2010; Font et al., 2010) provides the longest record for Sea Surface Salinity $\left(\mathrm{SSS}^{\mathrm{a}}\right)$ monitored from space over the global ocean (2010-present). The pioneered SMOS (2010-) and Aquarius (2011-2015) (Largeloef, 2008) satellite missions have demonstrated the capability of L-band radiometry for monitoring SSS from space (e.g. Reul et al., 2014a;

Lagerloef, 2012).

Salinity is a key ocean variable that plays a fundamental role in the density-driven global ocean circulation, the water cycle, and climate (Siedler et al., 2001). Salinity controls the density of sea water, together with temperature. At the ocean surface, in cold waters $(T=2$ ${ }^{\circ} \mathrm{C}$ ), a SSS change of $\sim 0.1 \mathrm{pss}^{\mathrm{b}}$ is equivalent, in terms of density, to a sea surface temperature (SST) change of $1^{\circ} \mathrm{C}$. SSS variations therefore greatly constrain the global thermohaline circulation as salinity drives the high latitude convective overturning. In warmer regions ( $\mathrm{T}=28^{\circ} \mathrm{C}$ ), a 0.44 pss change is equivalent to a $1{ }^{\circ} \mathrm{C}$ change in terms of density. Salinity stratification within a near isothermal layer (known as the barrier layer, e.g. Lukas and Lindstrom, 1991) can furthermore inhibit the vertical mixing of heat and momentum, and play a role in major phenomena such as the El Niño Southern Oscillation (e.g. Vialard and Delecluse, 1998), the southwest monsoon rain distribution (e.g. Shenoi et al. 2002) or the oceanic productivity (e.g. Picaut et al. 2001). Finally, SSS is considered as a passive tracer of

\footnotetext{
a SSS will hereafter refer to the salinity measured between $1 \mathrm{~cm}$-as monitored by satellite measurements- and at a few meters depth -as monitored by most in situ measurements. ${ }^{\mathrm{b}}$ pss is used here as an equivalent to gram of salt per kilogram of standard sea water, see UNESCO (1985) for more details
} 
the hydrological cycle, recording for instance its intensification in response to anthropogenic

64 climate change (e.g. Durack et al. 2012). For all these reasons, SSS has been designated as an ECV (Essential Climate Variable) by the Global Climate Observing System (GCOS).

SMOS data has enabled the study of salinity changes associated with two El Niño events (Hasson et al. submitted) and a La Niña event (Hasson et al. 2014), climate variability in the equatorial Indian Ocean (Durand et al. 2013), decadal salinity changes in the subtropical Pacific Ocean (Hasson et al. 2013) or North Atlantic Ocean (Grodsky et al. 2017). The spatial resolution and spatio-temporal coverage of the SMOS mission (50 km resolution; global coverage every 3 to 5 days) also allow the unprecedented detection of SSS mesoscale features associated with the transport across frontal regions (e.g. Reul et al., 2014b; Kolodziejczyk et al., 2015), very hardly accessible from Aquarius measurement (100-150 km resolution; global coverage every 8 days).

SMOS demonstrated performance in monitoring open-ocean salinity variations has been impressive so far. SMOS results have, however, been disappointing close to land, for instance in the Bay of Bengal, where Aquarius and more recently the Soil Moisture Active Passive (SMAP; 2015-) mission perform better (Akhil et al. 2016 and Fournier et al. 2017).

SMOS is an Earth Explorer mission. It carries an L-band Microwave Interferometric Radiometer with Aperture Synthesis (MIRAS), which is the first interferometer and the first L-band radiometer observing Earth from space. L-band $(1.4 \mathrm{GHz})$ is a passive protected frequency band but many SMOS measurements are corrupted by unexpected man-made Radio Frequency Interferences (RFI) (Oliva et al., 2012). SMOS SSS is also affected by the presence of nearby landmasses up to several hundreds of kilometers into the ocean, likely an effect of imperfect synthetic aperture image reconstruction in the present SMOS data 
processing (more on limitations in the present SMOS image reconstruction is presented in

87 Anterrieu et al., 2015).

Other two satellite missions measuring SSS from space, Aquarius (Lagerloef et al., 2008) (2011-2015) and SMAP (Piepmeier et al., 2017) (2015-present), are equipped with classical L-band radiometers. Hence, they are expected to suffer less land-sea contamination than SMOS. Aquarius and SMAP were launched subsequently to SMOS and have benefited from a better RFI-protected onboard processing.

The unique length of SMOS record and its high spatio-temporal resolution (comparable to the more recent SMAP mission) are strong motivations for improving its processing in order to mitigate RFI and land-sea contaminations on the retrieved SSS. The validation of satellite SSS using in situ SSS measurements is, however, very challenging in coastal areas where contaminations are strong, in situ data are very sparse and variability is high, such as in river plumes (Delcroix et al. 2005; Boutin et al. 2016). Hence, in addition to using in situ SSS, we take advantage of SMAP SSS to assess corrections to the SMOS SSS.

Kolodziejczyk et al. (2016) (K2016 hereafter) have developed a Bayesian methodology to mitigate SSS systematic errors due to land-sea contamination. The method is described in

102 detail in Section 3.3 of the present paper. It brings a clear improvement in most areas, with a 103 32\% decrease of the RMSD globally with respect to ship measurements. Some examples 104 below, however, indicate much lower SSS values in SMAP than in K2016 SMOS retrievals, 105 in particular near river mouths. In the Bay of Bengal, for instance, fresh water originating 106 from the Ganges-Brahmaputra (GB) is transported southward by the East India Coastal

107 Current (EICC) after the monsoon, forming a $200 \mathrm{~km}$ fresh water tongue along the Indian 108 coast, up to 10 pss fresher than in the central Bay of Bengal (Chaitanya et al. 2014). Fournier 
et al. (2017) demonstrated the SMAP capacity to monitor the modulation of this freshwater

110 tongue extent by climate variability and mesoscale eddies stirring the freshwater plume away

111 from the coast. This peculiar pattern is more than 3 pss fresher in SMAP SSS than SMOS

112 K2016 SSS (Figure 1 a and c). Fournier et al. (2016) similarly used SMAP data to study an

113 unusual freshening associated with anomalous advection of the Mississippi River plume in

114 the Gulf of Mexico. While this freshening is also detected by SMOS K2016 (Figure $1 \mathrm{~d}$ ), it is

115 saltier than in SMAP SSS (Figure $1 \mathrm{f}$ ). Such overestimation of K2016 SSS by SMAP relative

116 to SMOS in the low salinity regime also occurs in the eastern tropical Atlantic (Figure $1 \mathrm{~g}, \mathrm{i}$,

117 Congo and Niger river mouths, Reul et al. 2014a) and western tropical Atlantic (Amazon and

118 Orinoco, Figure $1 \mathrm{j}, \mathrm{l})$. SMOS K2016 default in retrieving the freshest SSS of the major river

119 plumes illustrates the need of an improved processing in variable, low-salinity regions near

120 land.

121 The purpose of this paper is to present a revised version of the K2016 methodology. The

122 main changes aim at taking the SSS natural variability into consideration in the land-sea

123 contamination correction and at adding a correction for the seasonally-varying latitudinal

124 biases.

125 Ancillary datasets are detailed in section II. An overview of the SMOS SSS retrieval, of the

126 K2016 SMOS processing and a description of the revised methodology are given in section

127 III. Comparisons with ancillary data sets are presented in section IV and V. They are

128 summarized and discussed in section VI. 

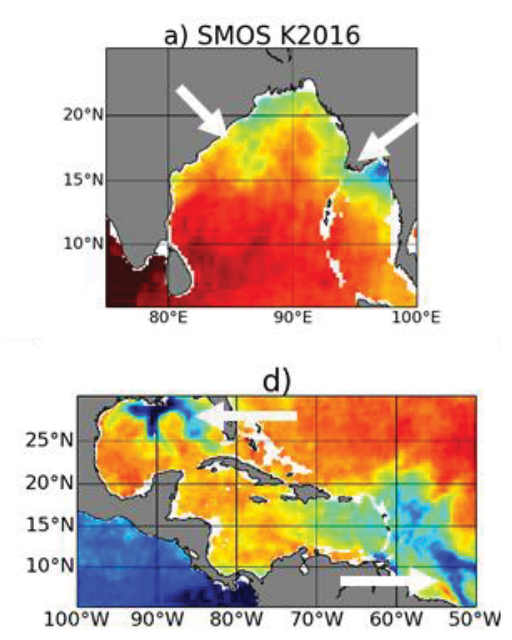

g)
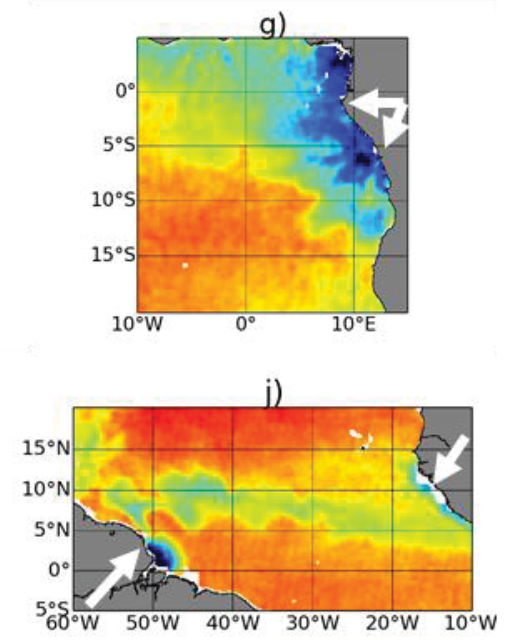

b) SMOS CEC

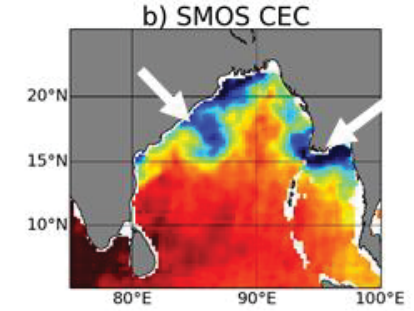

e)

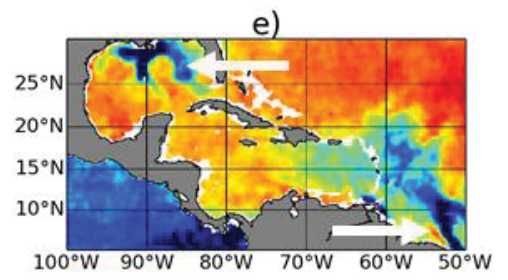

h)

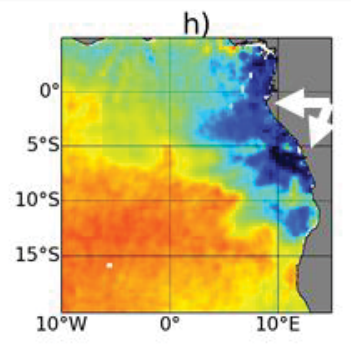

k)

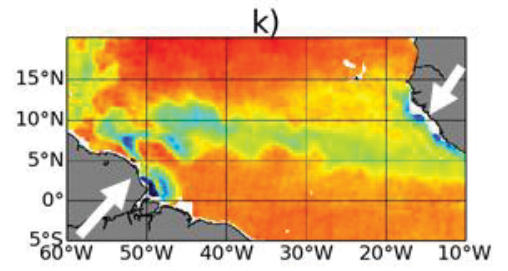

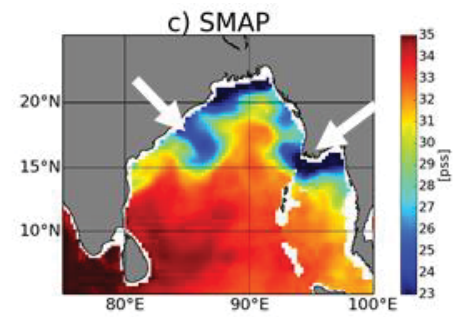
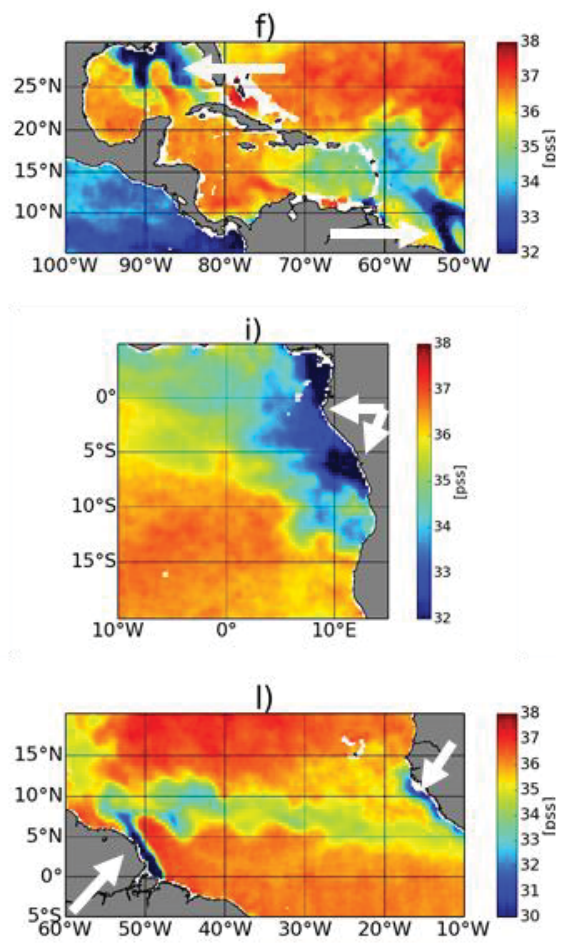

130 Figure 1: Satellite SSS: SMOS SSS corrected according to (a, d, g, j) K2016 methodology, (b, e, h, k)

131 the method described in this paper (CEC); (c, f, i, l) SMAP SSS. 4 case study areas : (a, b, c) : Bay of

132 Bengal - August 21 ${ }^{\text {st }}$ 2015; (d, e, f) : Gulf of Mexico - August18 ${ }^{\text {th }} 2015$; (g, h, i) : Eastern Tropical

133 Atlantic Freshwater Pools - April 14 $4^{\text {th }} 2016$; (j, k, l) : Amazon plume - October $21^{\text {st }} 2015$. SMOS and

134 SMAP SSS is averaged over a SMOS repetitive orbit sub-cycle (18 days) and two SMAP repetitive

135 orbit cycles (16 days) respectively. Striking fresh SSS features in better agreement with SMOS (new

136 version) and SMAP are indicated with white arrows.

Three types of ancillary data are used in this study. The In situ Analysis System (ISAS) SSS

140 is used both to set the long term mean reference of our correction and to qualitatively indicate

141 the most trustable SMOS SSS data in our correction process as described in Section 3. It is 
142 also used to check the SMOS SSS variability. SMAP and ship SSS are used for independent 143 assessment.

\subsection{In Situ Analyzed SSS}

145 Monthly gridded fields of salinity derived from in situ measurements are obtained from the

146 ISAS (In situ Analysis System) v6 algorithm, an optimal interpolation (Bretherton, 1976) tool

147 developed for the synthesis of the Argo global dataset (Gaillard et al., 2016). We use the

148 fields reconstructed at $5 \mathrm{~m}$ depth on a half degree horizontal grid. The ISAS Near Real Time

149 (NRT) products are available since 2010. In addition, over the 2002-2012 period, ISAS13

150 (Gaillard, 2015) fields have been produced after a refined quality check of the Argo profiles.

151 Data are preprocessed for ISAS13 using a climatological test and followed by a visual control

152 of suspicious profiles. The interpolation is based on delayed mode Argo floats, TAO-

153 TRITON-PIRATA-RAMA moorings and MEMO (Marine Mammals) data.

154 The ISAS-NRT fields (2010-present) are used by the correction method whereas ISAS13 (till 2012) and ISAS-NRT (from 2013 to 2016) fields are used for the assessment presented

156 Section 4.

\subsection{SMAP SSS}

158

The SMAP mission (Piepmeier et al. 2017) provides L-band radiometric observations since

April 2015. While its main objective is the observation of soil moisture, the observed brightness temperatures (Tb) are also used to retrieve SSS (Fore et al. 2016a). SMAP SSS

161 characteristics are quite close to those of SMOS in terms of spatio-temporal coverage and

162 spatial resolution ( $\sim 50 \mathrm{~km})$. In approximately 3 days, SMAP achieves global coverage and it has an exact orbit repeat cycle of 8 days. The SMAP L-band microwave radiometer, however, uses a conical scanning antenna instead of a synthetic aperture imaging antenna. As 
165 stated in the introduction, a particular attention was put on filtering the RFI (Mohammed et 166 al. 2016) and their impact is expected to be limited compared to SMOS. SMAP also suffers

167 from land-sea contamination but, given that SMAP carries a real aperture antenna, the

168 contamination is not expected to be as spatially variable as with SMOS. We use level 3

169 SMAP SSS produced at the Jet Propulsion Laboratory using the Combined Active Passive

170 (CAP version 3) algorithm (Fore et al. 2016b). A complete description of the CAP v3

171 algorithm can be found in Fore et al. (2016a), but a brief description follows. The CAP

172 algorithm is only applied to passive measurement as the radar failed a few months after

173 launch. It includes specific Tb corrections for land and galactic noise contaminations, and a

174 global Tb bias adjustment (latitude and time-dependent). After correction, the rms difference

175 of SMAP retrieved SSS with respect to Hycom SSS in the vicinity to land is less than 1.5pss.

176 Level 2 SSS is retrieved from SMAP Tb measurements using a constrained objective

177 function minimization. Data are mapped on a $0.25^{\circ}$ grid using a Gaussian weighting with a

178 search radius of approximatively $45 \mathrm{~km}$ and a half-power radius of $30 \mathrm{~km}$. They are

179 aggregated in level 3 maps produced daily with an 8-day running-average time window.

180 CAPv3 SMAP SSS agrees well with in situ SSS. Tang et al. 2017 found a rms difference of 0.26 pss between weekly SMAP SSS and buoy SSS. They also show that SMAP and SMOS SSS depict salinity fluctuations very close to in situ SSS.

\subsection{Ship SSS}

184 Salinity data provided by thermosalinographs (TSG) installed on voluntary merchant ships

185 are used as ground truth. A full description of the data can be found in Alory et al. (2015).

186 They provide SSS estimates with an $\sim 2.5 \mathrm{~km}$ resolution along the ship track and are

187 independent from the ISAS analyses. Samples are taken at a few meters depth. Noise on

188 individual ship SSS is estimated to be on the order of 0.08 pss (Alory et al. 2015). In the 
presence of strong vertical stratification, TSG and satellite SSS are expected to differ as the

190 L-band radiometer skin depth is about $1 \mathrm{~cm}$ (Boutin et al. 2016). This may occur under heavy

191 rain conditions or in river plumes. Because of their singular spatio-temporal resolution, ship

192 measurements, however, provide invaluable information on the spatial variability of SSS

193 unresolved by Argo.

\section{SMOS data and processing methodology}

195

196

197

198

200

201

202

203

204

205

206

207

208

209

210

211

212

The SMOS mission (Kerr et al., 2010) provides SSS measurements from space since January 2010. The SMOS satellite is on a sun-synchronous circular orbit with a local equator-crossing time at 6 AM on the ascending node and with a repeat sub-cycle of 18 days. It carries a 2-D interferometric radiometer, the MIRAS instrument. This groundbreaking technology was chosen as it involves much lighter antennas than real aperture antennas, and while getting ground spatial resolution on the order of $50 \mathrm{~km}$ at L-band frequency requires a huge antenna. The synthetic aperture antenna approach involves the reconstruction of an image using spatial Fourier components as derived from the correlations between numerous antenna elements (69 in case of SMOS). The SMOS bi-dimensional multi-angular images of $\mathrm{Tb}$ are reconstructed with a spatial resolution in the field of view ranging between about $35 \mathrm{~km}$ and $100 \mathrm{~km}$ (50 $\mathrm{km}$ on average). In this paper, we use the SSS retrieved within the center part of the field of view that extends at $+/-400 \mathrm{~km}$ away from the center of the satellite swath. Global ocean coverage is then achieved after about 5 days. Individual Tbs are very noisy (1.6-3.2 K) and lead to a typical noise on SSS of the order of 0.6 pss in tropical and subtropical regions on pixel-wise SSS retrievals (Hernandez et al., 2015; Supply et al., 2017). However, owing to the very good spatio-temporal coverage of SMOS, averaging SMOS SSS over typically one month and $100 \times 100 \mathrm{~km}^{2}$ results in an accuracy close to 0.2 pss in the open ocean, after removing a climatological mean of SMOS systematic errors (Boutin et al., 2016). 
213 In the following, before describing the new SSS correction methodology developed in the

214 present paper, we recall in section 3.1, the principle of the along track (level 2, L2) SMOS

215 SSS retrieval from Tb measurements, and, in section 3.2, the basis for the K2016 correction

216 method applied to L2 SSS.

\section{$217 \quad 3.1 \quad$ SMOS SSS level 2 retrieval}

218 The SMOS L2 SSS is retrieved from Level 1 (L1) Tb through a maximum-likelihood

219 Bayesian approach in which $\mathrm{Tb}$ measured in the antenna reference frame, $T b^{\text {meas }}$, are 220 compared with $\mathrm{Tb}$ simulated using a forward radiative transfer model, $T b^{\text {mod }}$ (see a general 221 description of the retrieval algorithm in Zine et al. (2008)). The retrieved parameters, $P_{i}$, and 222 their associated theoretical error, are estimated through the minimization of the $\chi^{2}$ cost 223 function:

$\chi^{2}=\sum_{n=1}^{N} \frac{\left[T b_{n}^{\text {meas }}-T b_{n}^{\text {mod }}\left(\theta_{n}, P_{i} \ldots\right)\right]^{2}}{\sigma_{T b n}^{2}}+\sum_{n=1}^{M} \frac{\left[P_{i}-P_{i 0}\right]^{2}}{\sigma_{P i 0}^{2}}$,

where $N$ is the number of measurements available for retrievals in vertical and horizontal polarizations at different incidence angles $\theta_{n}$. $\mathrm{N}$ is typically 120 to 240 within $+/-400 \mathrm{~km}$

227 from the center of the track. $\sigma_{\mathrm{An}}$ is taken equal to the SMOS brightness temperature noise 228 (between 1.6 and 3.2K depending on the location within the field of view) plus a small term that takes into account an error originating from the radiative transfer model error (see Zine et al. 2008 for more details). $M$ is the number of physical parameters, $P_{i}$ (SSS, wind, sea surface

231 temperature and ionospheric total electronic content) that are adjusted by the retrieval; $P_{i 0}$ and $\sigma_{\text {Pio }}$ are a priori values for $P_{i}$ and their associated errors respectively.

233 In the present study, we use SSS produced at the Data Production Center (CPDC) of the

234 Centre Aval de Traitement des Données SMOS (CATDS) in its RE05 version (Vergely and 
Boutin, 2017). Daily SSS fields are provided on a 25-km resolution EASE 2 (Equal-Area

236 Scalable Earth 2) grid (Brodzik et al. 2012) for ascending and descending orbits separately

237 (CATDS, 2017a). L1 Tbs, radiative transfer models (roughness model 1) and retrieval

238 scheme used in CATDS CPDC RE05 are identical to the ones used in the European Space

239 Agency level 2 ocean salinity processor version 622 (ESA L2OS v622) (see a description in

240 SMOS-Ocean Expert Support Laboratories (2014)). The main difference between the

241 CATDS RE05 and the ESA v622 processing involves the Tb outlier filtering. No Tb outlier

242 filtering is applied when retrieving SSS with ESA L2 OS V622. The absence of Tb outlier

243 filtering enables an easier detection of RFI-polluted SSS through a larger $\chi^{2}$ value (equation

244 1). This, however, removes pixels that are systematically contaminated by the presence of

245 nearby land, which could be mitigated by our correction. K2016 correction method was

246 indeed developed using ESA v5 processing in which an outlier filtering of $\mathrm{Tb}^{\text {meas }}$ was

247 performed and it was able to mitigate part of the RFI biases. In the CATDS RE05

248 processing, a $3 \sigma_{\mathrm{Tbn}}$ filtering is applied to $\left(\mathrm{Tb}^{\text {meas }}-\mathrm{Tb}^{\text {mod }}\right)$ before performing the SSS retrieval.

249 Some tests (not shown) performed on SSS retrieved from filtered and from non-filtered Tb

250 datasets confirm that the correction presented in this paper is more efficient when used in

251 conjunction with a Tb filtering.

\subsection{K2016 land-sea contamination correction}

254 In this section, we briefly review the K2016 methodology. The K2016 correction aims at 255 mitigating systematic errors constant with time and was shown to efficiently correct land-sea contamination in many regions. Given the 18-day sub-cycle of SMOS, a given location over

257 the ocean is observed with the same SMOS measurement geometry every 18 days; within 
18 days, it is sampled by several SMOS SSS measurements which are located at various

259

260

261 locations across the swath, $\mathrm{x}_{\text {swath }}$. The K2016 methodology considers that the long term (2010-2014) SSS variability observed by SMOS has to be rather similar whatever $\mathrm{x}_{\text {swath }}$ and the orbit orientation $\mathrm{x}_{\text {orb }}$. Relative biases, bland, with respect to a reference SSS, SSS $_{\text {ref, }}$ are derived from SMOS SSS through a least square minimization approach, and through a series

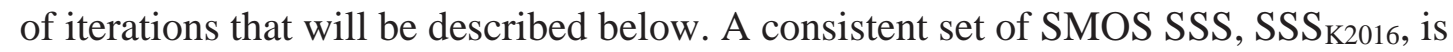
obtained as:

$$
\operatorname{SSS}_{\mathrm{K} 2016}\left(\mathrm{t}, \phi, \lambda, \mathrm{X}_{\text {swath }}, \mathrm{X}_{\mathrm{orb}}\right)=\mathrm{SSS}_{\mathrm{ref}}(\mathrm{t}, \phi, \lambda)-\mathrm{b}_{\text {land }}\left(\phi, \lambda, \mathrm{x}_{\mathrm{swath}}, \mathrm{x}_{\mathrm{orb}}\right)
$$

where $t$ is the time of the measurement, $\phi$, and, $\lambda$, are respectively the latitude and the longitude of the considered location over the ocean. $\mathrm{x}_{\text {swath }}$ is sampled within $25 \mathrm{~km}$ wide bins. $\mathrm{b}_{\text {land }}$ and $\mathrm{SSS}_{\text {ref }}$ are derived as follows. Defining $\mathrm{p}=\left(\mathrm{SSS}_{\mathrm{ref}}, \mathrm{b}_{\text {land }}\right)^{\mathrm{T}}$, $\mathrm{p}_{0}$ the a priori values of $\mathrm{p}$, $\mathrm{y}_{0}$ the SMOS SSS, the estimated values of $\mathrm{p}$, pest, are derived as:

$$
p_{e s t}=p_{0}+C_{p} \cdot G^{T} \cdot\left(G \cdot C_{p} \cdot G^{T}+R\right)^{-1} \cdot\left[y_{0}-f\left(p_{0}\right)\right]
$$

where $\mathrm{G}$ is the matrix of derivatives of observations with respect to the parameters (also called observational operator), $\mathrm{R}$ is the covariance matrix for the observation error, $\mathrm{C}_{\mathrm{p}}$ is the covariance matrix for the a priori error on the parameters p. $C_{p}$ is parametrized as a function of an acceptable standard deviation of SSS, $\sigma_{\text {ssSref, }}$ over a correlation timescale $\tau$.

The minimization is repeated four times, twice with $\tau=16$ days (corresponding to a 18-day Gaussian smoothing window), then twice with $\tau=8$ days (corresponding to a 9-day Gaussian smoothing window). At each iteration, a new set of a priori values for $\mathrm{p}$ and for $\sigma_{\text {sssref }}$ are computed. During the first iteration, the a priori values of $\mathrm{SSS}_{\text {ref, }} \mathrm{SSS}_{\text {refo, }}$ are taken as the median of SMOS SSS at the center of its swath over the 2010-2014 period, the a priori value 
of bland is equal to 0 , $\sigma_{\text {sssref }}$ is taken equal to 0.3 pss, and the observation errors are taken equal to the theoretical error associated with the L2 SMOS SSS retrieval, Esss_L2. SSS bland1 are computed from the p and $\sigma_{s s s r e f}$ solutions of the first iteration. During the second 283 iteration, SSS outliers, linked primarily to RFI contamination, are detected using a 3-sigma

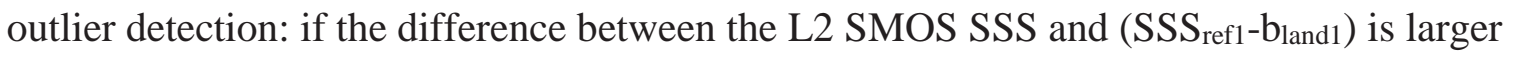
than 3 times Esss_L2, the error on the measurement indicated in the matrix R is artificially

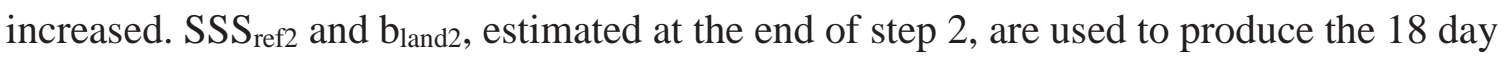

287 SSS K2016 fields. The third and fourth iterations aims at optimizing SSS $_{\text {ref }}$ and $b_{\text {land }}$ at 9 day resolution. During the third iteration, $\mathrm{SSS}_{\text {ref2 }}$ and $\mathrm{b}_{\text {land2 }}$ are taken as a priori parameters, $\tau$ is reduced to 8 days and $\sigma_{\text {ssSref }}$ is increased to 0.5 pss resulting in SSS $_{\text {ref3 }}$ and bland3. The fourth step leading to $\mathrm{SSS}_{\text {ref4 }}$ and $\mathrm{b}_{\text {land4 }}$ is similar to the second one using the same a priori values as in step 3. At the end, an additional term is added to the estimated bias, to ensure that the 4-

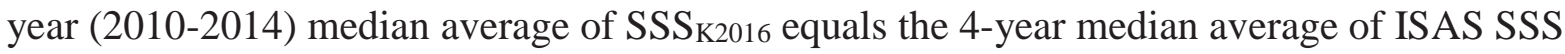
for each latitude and longitude:

with $b_{\text {landx }}$ equals to bland2 in the case of 18-day corrected field estimates, or to bland4 in the case of 9-day corrected fields. Note that the last term of Equation (4) is the only external

297 information used in the entire correction process and does not modify the temporal variability 298 of the observed fields.

The K2016 methodology was developed based on SMOS SSS processed with ESA L2OS

300 version 550. In order to provide consistent comparison of the K2016 corrected SSS

301 (SSS K2016 $)$ and the newly corrected dataset presented in this paper (SSS J2018), SSS $_{\mathrm{K} 2016}$ was re302

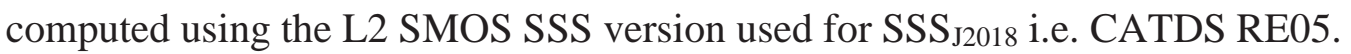




\subsection{New correction}

304 In the present paper, we add a correction for seasonally-varying latitudinal biases, $b_{\text {lat, }}$ and we

305 update the land-sea contamination correction, $b_{\text {land, }}$ with respect to K2016. $b_{\text {lat }}$ and $b_{\text {land }}$ are

306 assumed to be additive, so that the corrected SSS, SSS $_{J 2018}$, is expressed as:

$307 \operatorname{SSS}_{\mathrm{J} 2018}\left(\mathrm{t}, \phi, \lambda, \mathrm{X}_{\text {swath }}, \mathrm{X}_{\mathrm{orb}}\right)=\mathrm{SSS}_{\text {ref }}(\mathrm{t}, \phi, \lambda)-\mathrm{b}_{\text {land }}\left(\phi, \lambda, \mathrm{X}_{\mathrm{swath}}, \mathrm{X}_{\mathrm{orb}}\right)-\mathrm{b}_{\text {lat }}\left(\phi, \mathrm{X}_{\text {swath }}, \mathrm{x}_{\mathrm{orb}}, \mathrm{m}\right)$

308 where $\mathrm{m}$ is the month of the SMOS pass. In a last step, similar to K2016 (equation 4), the 7-

309 year (2010-2016) median average of the corrected SSS is adjusted, for each latitude and

310 longitude, to the 7-year median average of ISAS SSS. The latter is the only quantitative

311 information external to SMOS data used in the correction process and does not modify the

312 temporal variability to the observed fields.

\subsubsection{Observed seasonally-varying latitudinal biases}

314 Further than $1000 \mathrm{~km}$ from the coastline, land-sea contamination is not detectable but

315 seasonally-varying latitudinal biases are observed. They mostly depend on $\mathrm{X}_{\text {swath }}$, $\mathrm{x}_{\text {orb }}$, and the

316 month of the year. The two examples on Figure 2 illustrate the behavior for two extreme

317 cases. In November (Figure 2 a-c), in the center of the swath, SMOS SSS latitudinal

318 variations are very close to ISAS SSS latitudinal variations on ascending orbits but not on

319 descending orbits. In January (Figure 2 b-d), descending orbits at the edge of the swath

320 display strong biases with respect to ISAS while ascending orbits do not. The systematic

321 errors are quite stable from year to year, as indicated by the standard deviation of the 2011 to

3222016 monthly latitudinal SMOS minus ISAS SSS difference (Figure 2c and d). It is not true

323 at high latitudes where, in most cases, both the mean and standard deviation of the

324 differences are high. This is likely associated with an effect of ice contamination. Systematic

325 errors observed over other ocean basins are similar (see Appendix A1). These systematic 


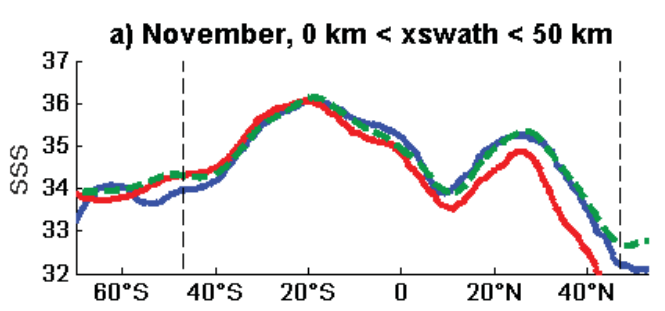

c)

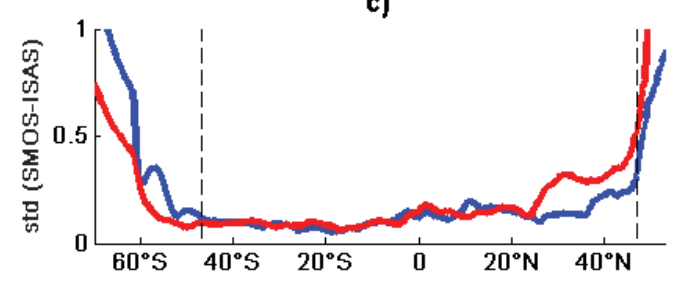

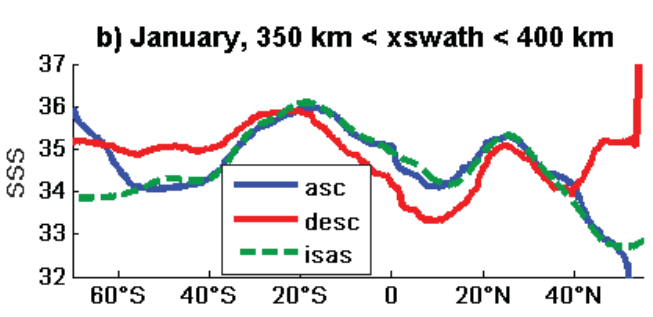

d)

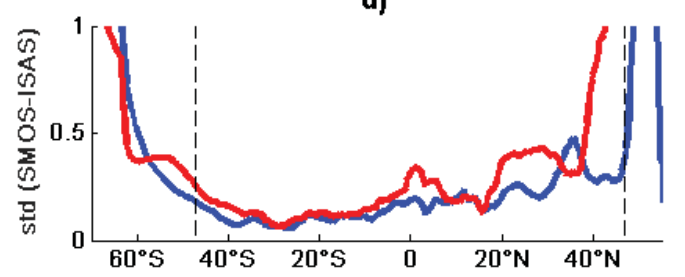

Figure 2 : Two examples of 2011-2016 latitudinal profiles of mean SSS (a; b) and of the standard deviation of the 2011-2016 monthly differences between SMOS SSS and ISAS SSS (c; d). The latitudinal means and standard deviations are computed over the Pacific Ocean further than $1200 \mathrm{~km}$ from any coast: green: ISAS, blue: SMOS ascending orbits; red: SMOS descending orbits: a;c) November; middle of the swath (0-50 km from the center of the swath); b; d) January; edge of the swath (350-400 km from the center of the swath). Dashed vertical lines indicate $47^{\circ} \mathrm{N}$ and $47^{\circ} \mathrm{S}$.

\subsubsection{Correction for seasonally-varying latitudinal biases}

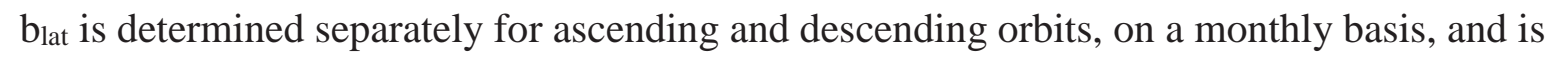
assumed to be independent of the longitude and of the year. We neglect interannual variations that could result from variation in sun activity, as they appear to be an order of magnitude smaller than the seasonal biases (see section 3.3.1). The correction is estimated from Pacific Ocean orbits further than $1200 \mathrm{~km}$ from continental coasts, in order to avoid land-sea contamination (bland in Eqn 4 vanishes in this case) and because the northern latitudes in the Pacific Ocean are less affected by RFI than in the Atlantic Ocean. For $\mathrm{X}_{\text {swath }}$ locations and seasons not very affected by RFI at high latitudes, we checked that biases are similar in the 
345 Pacific and Atlantic Ocean (see Appendix A1). For each $\mathrm{x}_{\text {swath }}$ and $\mathrm{x}_{\text {orb }}$, twelve sets of

346 monthly latitudinal corrections are estimated by comparing SMOS SSS on contaminated and

347 non-contaminated $\mathrm{x}_{\text {swath }}$ intervals. The first step is to choose a set of non-contaminated $\mathrm{x}_{\text {swath }}$

348 for each month and for each $\mathrm{x}_{\text {orb }}$ that is used as reference in our correction methodology. The

349 non-contaminated $\mathrm{x}_{\text {swath }}$ locations are identified from comparisons between 6-year averaged

350 (2011-2016) monthly latitudinal SSS profile at $0.25^{\circ}$ resolution derived for each SMOS $\mathrm{x}_{\text {swath }}$

351 location and from ISAS as described in Appendix A1. The 2010 year is not considered for the correction estimate as the calibration of the MIRAS instrument was not very stable during the SMOS commissioning period (January to June 2010). The latitudinal profiles of the unbiased SMOS SSS at reference $\mathrm{x}_{\text {swath }}$ locations determined for a given month, are averaged together to provide a reference SSS latitudinal profile. The latitudinal correction is then estimated as the median difference, per $5^{\circ}$ latitude, over the EASE2 grid latitudinal sampling, between the latitudinal profiles of the SMOS SSS at contaminated $\mathrm{x}_{\text {swath }}$ and the reference SSS latitudinal profile. The SMOS SSS latitudinal profiles differ from the ones based on ISAS SSS at high latitudes (Figure 2). This difference may be explained by remaining RFI contamination in the

360 northern latitudes but also by sea-ice contamination extending equatorward to about $1000 \mathrm{~km}$

361 from the ice edge. On ascending and on most descending latitudinal profiles, large differences between SMOS and ISAS SSS are indeed found poleward of $47^{\circ} \mathrm{N}$ (see two examples on Figure 2). Some degradation also occurs between $40^{\circ}$ and $47^{\circ} \mathrm{N}$ (see a worse case on Figure 2d). It concerns only a few $\mathrm{X}_{\text {swath }}$ and months on descending orbits and is

365 therefore rather limited. In the Southern Ocean, in Spring and Summer (Figure 2a-b), large differences only appear way south of $47^{\circ} \mathrm{S}$. However, in Winter, especially in the Atlantic

367 Ocean where the ice edge can be as north as $55^{\circ} \mathrm{S}$, large differences can reach $47^{\circ} \mathrm{S}$. As a 
compromise, in the following, the correction is applied only to latitudes within $47^{\circ} \mathrm{S}-47^{\circ} \mathrm{N}$ and results will be limited to this latitudinal range.

\subsubsection{Updated land-sea contamination correction}

371

372

373

375

376

377

378

379

380

381

382

383

384

385

386

387

388

389

Before estimating the land-sea contamination correction, we apply seasonally-varying latitudinal corrections determined as described in the previous section. Actually, an imperfect correction of sun and galactic noise effects is expected to generate systematic seasonal biases whatever the distance to the coast.

With respect to K2016, we make the following changes:

- In K2016, the covariance matrix of observation error, R, was filled with ESSS_L2 times the Identity matrix. With this approach, the observation errors depend only on the Jacobian of the modelled Tbs with respect to the retrieved parameters, on the a priori error on SMOS Tbs (equal to the SMOS radiometric noise) and on the a priori errors on auxiliary parameters. It does not take into account the actual differences between SMOS observed and modelled Tbs. In most cases, this difference is very close to the radiometric noise (e.g. Yin et al. 2012) and the associated $\chi$ (equation 1) normalized by the root mean square of $\mathrm{N}, \chi_{\mathrm{N}}$, is close to 1 . However, in case of polluted areas (e.g. RFI), $\chi_{\mathrm{N}}$ becomes larger than 1 . In the updated method, the errors specified in $\mathrm{R}$ are set to $\left(\mathrm{E}_{\mathrm{SSS} \_\mathrm{L} 2} \cdot \chi_{\mathrm{N}}\right)$ in order to take observed mismatches between SMOS measured and modelled Tbs into account. In case $\chi_{\mathrm{N}}$ is greater than 3, the particular SMOS SSS retrieval is not used in the correction estimate. 
- In K2016, osssref was a fixed value (0.3 pss for $\tau=16$ days; 0.5 pss for $\tau=8$ days). GssSref now uses an estimate of the SSS natural variability standard deviation, $\sigma_{\text {sssnat, }}$ as derived from SMOS measurements themselves. We derive $\sigma_{\text {ssSnat }}$ using a two-step iterative procedure, in which we first compute debiased SSS using $\sigma_{\text {SSSref }}=0.3$ pss for each grid point over the whole period as before, then we recompute debiased SSS using $\sigma_{\text {SSSref }}$ equal to the standard deviation of the debiased SSS from step 1. OssSnat is taken as the standard deviation of the debiased SSS obtained in step 2. In the open ocean $\sigma_{\text {ssSnat }}$ is 398 very close to the value we used in the previous version (0.3 pss) (Figure $3 \mathrm{a}$ ), but it is much larger in regions characterized by large inputs of freshwater, such as river plumes (e.g. Amazon plume, Bay of Bengal, Gulf of Mexico), rainy areas (e.g. Intertropical Convergence Zone, eastern and western tropical Pacific fresh pools) and areas characterized by numerous mesoscale features (e.g. Gulf Stream, south east of the Arabian Sea). With this variable $\sigma_{\text {ssSref }}$ we 404 allow SSS $_{\text {ref }}$ to vary more temporally in high variability regions through equation (3).

- The biases are derived from 7 years (2010-2016) of SMOS data instead of 4 years in K2016.

408

409

410 
a)

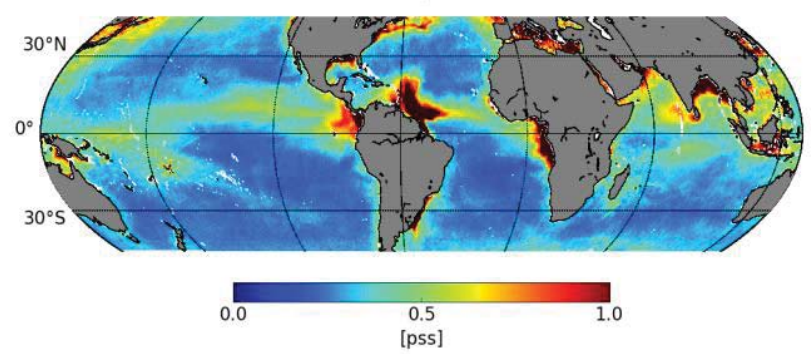

b)

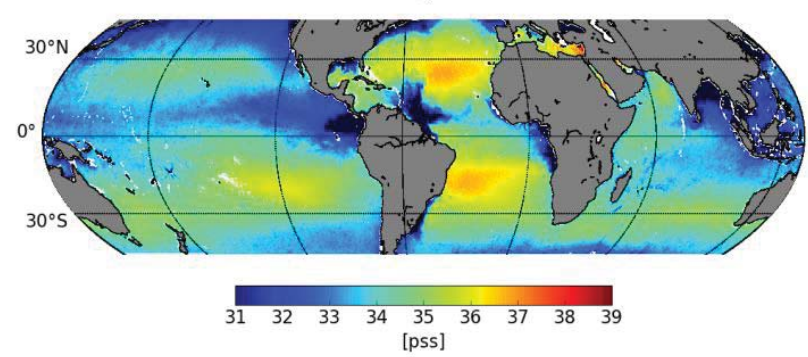

C)

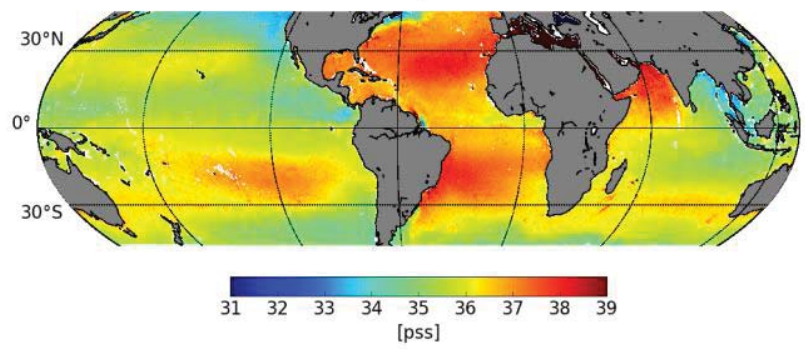

413 Figure 3 : a) SSS variability ( $\sigma_{\text {ssSnat }}$ ) derived from 7 years of SMOS filtered and corrected SSS (after 414 debiasing and filtering): large values are observed in river plumes and in rainy areas (ITCZ, SPCZ. b) 415 Minimum and c) maximum of the SSS as derived from 18-day CEC LOCEAN that are used in the 416 mapping of debiased near-real time products (see section 3.4).

\subsection{Mapping methods}

419 All SMOS level 3 maps shown in this paper include only SSS retrieved under moderate wind speed (3-12 $\left.\mathrm{m} \mathrm{s}^{-1}\right)$ and within +/-400 km from the center of the swath.

421 The non-bias corrected SMOS SSS is taken from the CATDS CPDC RE05 default

422 processing. Daily SMOS SSS retrieved over ascending and descending orbits are combined 423 to produce level 3 fields (L3P) (CATDS, 2017b). L3P fields over a 25x25 km² EASE 2 grid 
424 are obtained from SMOS SSS weighted by EsssL2 and averaged within monthly and $~ 10$ 425 days $25 \times 25 \mathrm{~km}^{2}$ bins. Measurements are filtered based on SSS retrieval quality flags and 426 avoiding regions suffering from major contaminations on Tb (e.g. galactic noise). A full 427 description of the procedure is available in Vergely and Boutin (2017).

428 Two sets of level 3 bias-corrected SMOS SSS fields are considered in this paper. The same 429 biases are applied (equation 5) but the filtering and mapping methods are different, partly due 430 to operational constraints in CATDS CPDC processing. One set, named L3Q, is processed in 431 near real time by the CATDS CPDC operational chain using a mapping procedure similar to 432 the one applied to L3P products. The other set, named CEC, is processed in delayed time by 433 the LOCEAN expertise center (CEC) of CATDS with a filtering and mapping procedure 434 similar to K2016. Hence, in the result section, changes brought by our new correction with 435 respect to non-corrected SSS will be evaluated by studying L3P and L3Q fields. Changes 436 with respect to K2016 methodology will be evaluated by studying K2016 and CEC fields. 437 The main characteristics of the L3P, K2016, CEC and L3Q processing are summarized in 438 Table 1. 

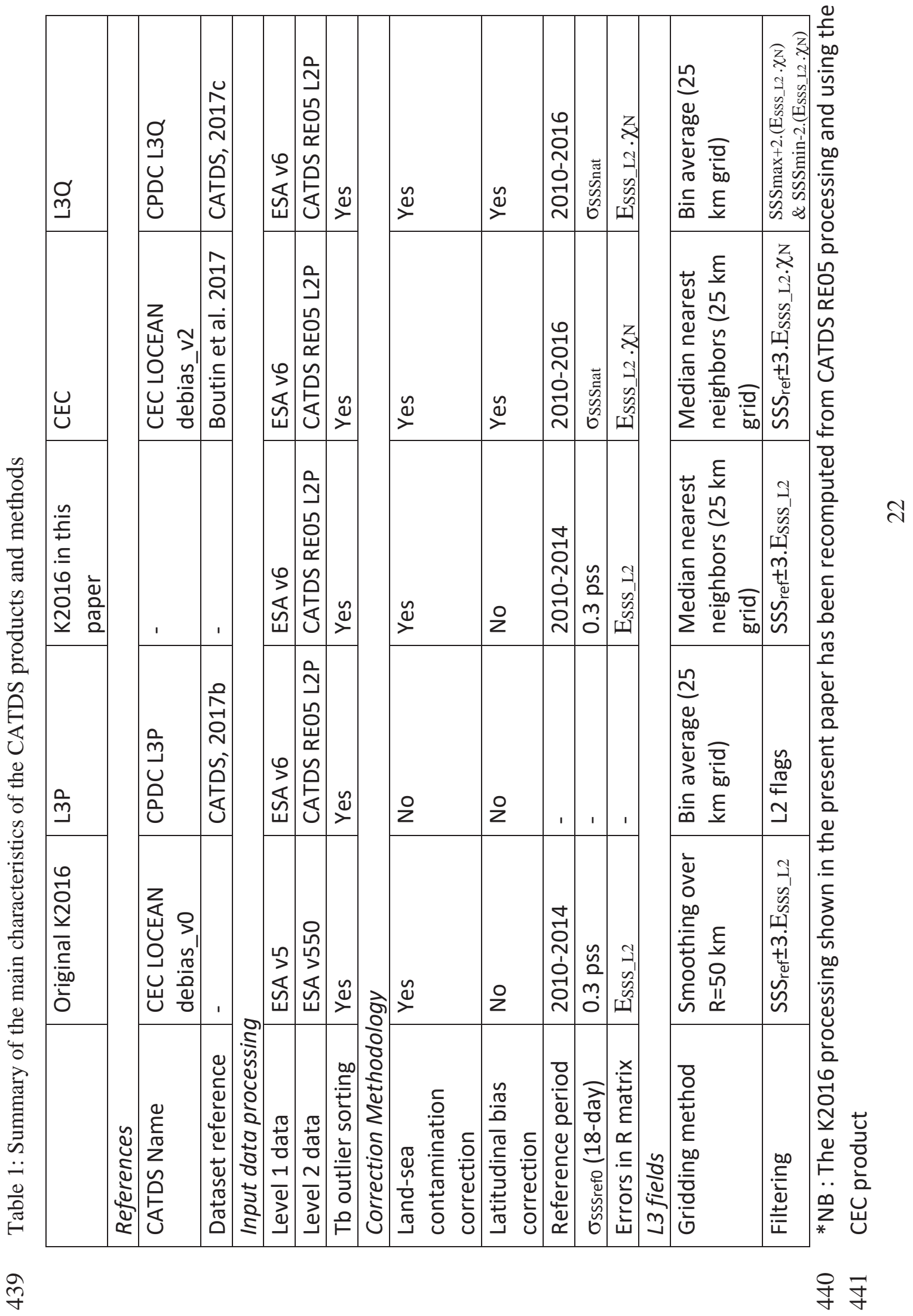
442 We now describe in detail the mapping and filtering procedures for generating L3P and CEC 443 fields:

- At the CATDS CEC LOCEAN, SSS gridded fields at $25 \times 25 \mathrm{~km}^{2}$ resolution, named CEC SSS in the rest of the paper, are built from the combination of debiased SSS which have been filtered from outliers in the course of the biases estimates (see description of steps 2 and 4 in K2016 methodology (section 3.2)). Debiased SSS are temporally averaged using a convolution with a Gaussian kernel with a full width of either 9 or 18 days at half maximum. In addition, a median filtering over nearest neighbors is applied to reduce remaining noise. CEC fields are built every 4 days over the 2010-2016 period (Boutin et al. 2017). From the 18-day CEC SSS fields over the 2010-2016 period, a minimum (SSSmin) and maximum (SSSmax) SSS is estimated at each grid point (Figure 3b and c) and is used to filter the operational CATDS CPDC products (see below).

- The CATDS CPDC operational chain provides near-real time data, at the expense of a less-refined data filtering. Biases are estimated as described previously and are applied (equation 4) to daily L3P SSS. For each orbit orientation, we define upper and lower acceptable bounds for daily SSS, based on acceptable absolute values and on SSS natural variability. The upper bound is the minimum value between 40 pss and SSSmax+2.(Esss_L2 $\left.\cdot \chi_{\mathrm{N}}\right)$; the lower bound is the maximum value between 5 pss and SSSmin-2.(ESSS_L2 $\left.\cdot \chi_{\mathrm{N}}\right)$. SSS with (Esss_L2 $\chi_{\mathrm{N}}$ ) larger than 3 pss are filtered out. Level 3 SSS fields, named L3Q in the rest of the paper, are then obtained using a simple average of the SSS weighted by (Esss_L2 $\chi_{\mathrm{N}}$ ) over one month or $~ 10$-day. A full description 

of the procedure is available in Vergely and Boutin (2017). Corrected fields are produced in near-real time at various spatial resolution (CATDS, 2017c). In this paper we use the $25 \mathrm{~km}$ resolution products.

\section{Comparison to ISAS}

Before assessing the new CEC and L3Q SSS fields with products which are not used in the correction method, we compare the corrected and non-corrected SMOS SSS fields with ISAS SSS fields. The comparison is restricted to L3P and L3Q SMOS SSS fields because these two fields are mapped using the same methodology.

474 Even if ISAS SSS is used as a guide to choose the reference $\mathrm{x}_{\text {swath }}$ in the latitudinal correction, we recall that the only quantitative ISAS information entering our method is the 7-year median average of the ISAS SSS fields. The amplitude of temporal variability is independent of ISAS SSS variability. It is thus informative to compare the SSS temporal variability detected by SMOS and ISAS.

479

By construction, the 7-year mean SMOS minus ISAS SSS difference is expected to be small. It is nevertheless non-zero everywhere as we apply a more stringent filtering in the course of the correction estimate than in the L3Q bin average computation. At less than $800 \mathrm{~km}$ from coasts, the mean difference between SMOS SSS and ISAS SSS is reduced from -0.5 pss to 0.07 pss (Table 2). The remaining -0.07 pss difference is likely due to the lack of in situ measurement in very fresh areas in the vicinity of land (less than 2000m depth) and to nonGaussian short-scale SSS variability smoothed out by ISAS objective mapping. In addition, SMOS samples the very near surface measurement $(\sim 1 \mathrm{~cm})$ while most in situ measurements used in ISAS analysis are performed close to 5m depth (Boutin et al. 2016). The standard 
deviation of the differences (Figure $4 \mathrm{a} \& \mathrm{~b}$ ) is much reduced in the vicinity of continents, except in river plumes areas but there, it could be an effect of ISAS smoothing.

Table 2: Statistics of monthly SMOS SSS (only pixels with more than 8 SMOS SSS retrievals in ascending and descending orbits are considered) minus ISAS SSS ; 2010-2016

\begin{tabular}{|l|l|l|l|}
\hline & $\begin{array}{l}\text { Number of } \\
\text { pixels }\end{array}$ & Mean bias (SMOS - ISAS) & std(SMOS-ISAS) \\
\hline $45^{\circ} \mathrm{S}-45^{\circ} \mathrm{N}$ distance to coast $<800 \mathrm{~km}$ & 0.63 \\
\hline $\begin{array}{l}\text { SMOS without } \\
\text { correction (L3P) }\end{array}$ & 1542456 & -0.53 & 0.49 \\
\hline $\begin{array}{l}\text { SMOS with correction } \\
(\mathrm{L} 3 \mathrm{Q})\end{array}$ & 1917346 & -0.07 & 0.26 \\
\hline \begin{tabular}{l}
$45^{\circ} \mathrm{S}-45^{\circ} \mathrm{N}$ distance to coast $>800 \mathrm{~km}$ \\
\hline $\begin{array}{l}\text { SMOS without } \\
\text { correction (L3P) }\end{array}$
\end{tabular} & 5316809 & -0.10 & 0.20 \\
\hline $\begin{array}{l}\text { SMOS with correction } \\
\text { (L3Q) }\end{array}$ & 5429659 & -0.02 & \\
\hline
\end{tabular}

492

493 In order to more precisely quantify the improvements between the L3Q and L3P SMOS SSS,

494 we detect the number of months, N, between July 2010 and December2016, for which the

495 absolute value of the difference between the L3Q and the L3P SSS is larger than a threshold,

496 T equal to 0.2 pss (Figure 4).
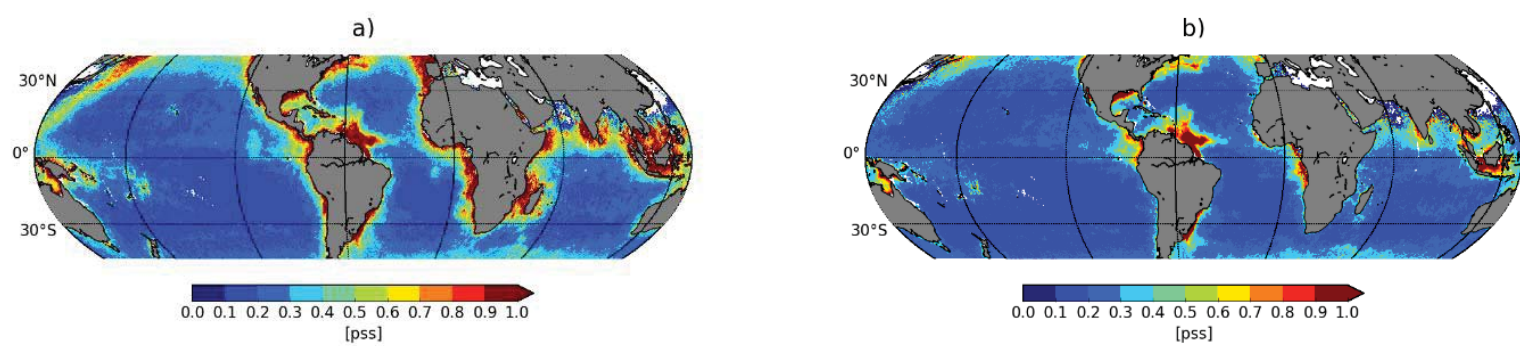

c)
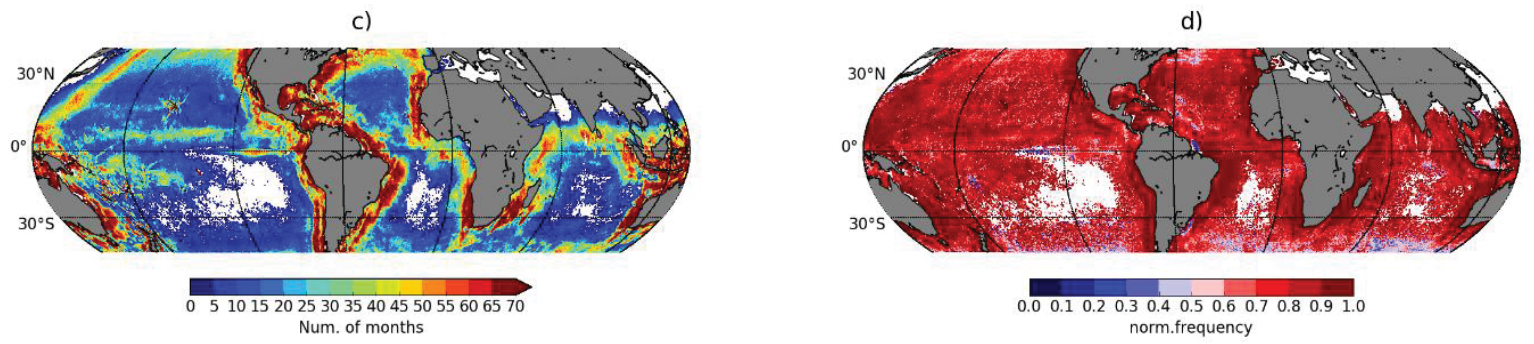
Figure 4 : Monthly SMOS SSS compared to monthly ISAS SSS from July 2010 to December 2016. Standard deviation of the differences for a) L3P SMOS SSS; b) L3Q SMOS SSS. c) Number of months with differences between L3P and L3Q SMOS SSS greater than 0.2 pss. d) Frequency with which corrections identified on Figure c) correspond to decreased bias with respect to ISAS (i.e. L3Q SMOS SSS closer to ISAS SSS than L3P SMOS SSS): red color means that the correction improves most of the time; blue color means that the correction degrades most of the time. Blank colors in figures c) and d) mean no change above the 0.2 pss threshold or no data in the L3P version (the comparison is done only for valid L3P SSS).

As expected, the number of months affected by the correction in a given pixel is higher in the vicinity of continents. In a next step we evaluate how frequently the changes correspond to improvements. For these months significantly affected by the correction, we thus compute the number of months with L3Q SSS closer to SSSisas than to L3P SSS. In most areas, the correction brings monthly SMOS SSS closer to monthly ISAS SSS in $60 \%$ to $100 \%$ of the cases (Figure $4 \mathrm{~d}$ ). This is not true in the Gulf Stream region close to $40^{\circ} \mathrm{N}$, probably because ISAS is not able to reproduce SSS mesoscale variability recorded by SMOS (Reul et al. 2014b), nor close to $10^{\circ} \mathrm{S}$ in the western Pacific Ocean and in the middle Indian Ocean, two regions strongly affected by RFI. It is nevertheless remarkable that other regions affected by RFI such as the north-western Pacific Ocean are improved most of the time, suggesting that the RFI disturbances there are sufficiently stable in time to be partly mitigated by our correction.

\section{Assessment of the corrected fields}

\subsection{Comparison to SMAP SSS}

SMAP CAP SSS has a similar spatial resolution as SMOS CEC SSS, SMAP passes are at 6AM and 6PM local time like SMOS, so that the spatio-temporal sampling of SMOS and SMAP are really comparable. SMAP SSS are much better filtered from RFI, hence providing an unprecedented monitoring of main river plumes in the vicinity of continents. On the other 
hand, SMAP Tb calibration is more challenging than for AQUARIUS (Fore et al. 2016a), so that the absolute value of SMAP SSS may remain imprecise to about 0.2 pss in low to midlatitudes of the open ocean, but biases up to 0.45 pss, which origin remains unclear, have also been reported during certain periods in the Bay of Bengal (Tang et al. 2017, their Figures 5 and 12 respectively). It is out of the scope of this paper to study SMAP CAP SSS biases. We

529 focus the investigation on the SSS variability measured by both sensors.

The various SMOS SSS fields are compared with SMAP SSS fields over the period between April 2015 and December 2016. Two ranges of temporal resolutions are considered, one close to one week, another one close to 18 days. The choices of the average durations are guided by the satellite repetitive orbit cycle and sub-cycle in order to get, for each instrument, the most even spatial coverage. In the following, for simplicity, 10-day L3P, L3Q and 9-day CEC SMOS SSS fields compared with 8-day SMAP SSS fields are referred to as 'weekly' comparisons. Comparisons between 18-day SMOS SSS fields from K2016 and CEC processing with 16-day SMAP SSS are referred to as 'bi-weekly' comparisons. We always compare fields centered on the same time (at \pm 12 hours), in order to minimize the effect of the different durations.

At global scale and 'weekly' resolution (Figure 5), standard deviations of the SMOS minus SMAP SSS differences are reduced in the vicinity of large continents and of RFI sources (e.g.

542 Fiji island, Hawaï island, south of Madagascar) from more than 0.6 pss before correction

543 (L3P, Figure 5 b) to less than 0.4 pss after correction (L3Q, Figure 5 e; CEC, Figure 5 h)

544 becoming comparable to open ocean values. In addition, the number of valid pixels is

545 increased, especially in the vicinity of large continents (Figure 5 c, $\mathrm{f}$ and i). The improvement

546 is better with CEC fields than with L3Q fields due to the improved filtering. The square of

547 the Pearson correlation coefficient, $\mathrm{r}^{2}$, is as good or better when considering L3Q instead of 

that is explained by SMOS SSS. Hence, if the natural SSS variability is low relatively to the satellite SSS noise, $\mathrm{r}^{2}$ is expected to remain small whereas if the natural variability is large compared to the satellite SSS noise, $r^{2}$ is expected to increase. This is what is observed. $r^{2}$ is in particular increased from less than 0.5 to above 0.5 in the north of the Gulf of Mexico, in the Gulf of Guinea, in the Bay of Bengal (no valid measurements exist there in the L3P processing) and to the north of the Amazon plume. The improvement is even larger when considering CEC SSS (Figure 5 i) instead of L3Q SSS due to the different filtering and mapping procedures: then, $\mathrm{r}^{2}$ in the above-identified regions becomes higher than 0.8 . These large values of $r^{2}$ correspond to regions of large natural SSS variability, much larger than the SSS noise, as will be shown below. On the other hand, in most regions of the open ocean where SSS variability is on the same order or smaller than SSS noise, $\mathrm{r}^{2}$ remains small. If instead of considering all the available SMOS SSS pixels (Figure 5), the comparison is made using only SSS pixels available in every SSS products (Appendix A2), the standard

562 deviations of the differences are comparable or slightly lower in regions polluted by RFI but

563 this is at the expense of many measurements which contain meaningful variability as

564 indicated by high $\mathrm{r}^{2}$ on Figure 5. Figure 5 indicates a clear improvement of L3Q and CEC fields with respect to L3P fields. In comparison with K2016 (not shown), standard deviations of the SMOS CEC 18-day SSS

567 minus SMAP SSS differences are very similar (within +/-0.05 pss) in major parts of the 568 ocean, but in the regions identified above where $\mathrm{r}^{2}$ became larger than 0.8 , they are locally improved by more than 0.5 pss; these regions are further studied below. We observe some degradation (standard deviations of the SMOS minus SMAP SSS differences increase by up to 0.3 pss) in some regions (the Mediterranean Sea, the Arabian Sea, the north-western part of 
572 the Pacific Ocean) strongly affected by RFI and for which L3P fields do not provide valid 573 measurements. In these regions, however, $\mathrm{r}^{2}$ obtained with both CEC and K2016 versions 574 remain less than 0.2 . 

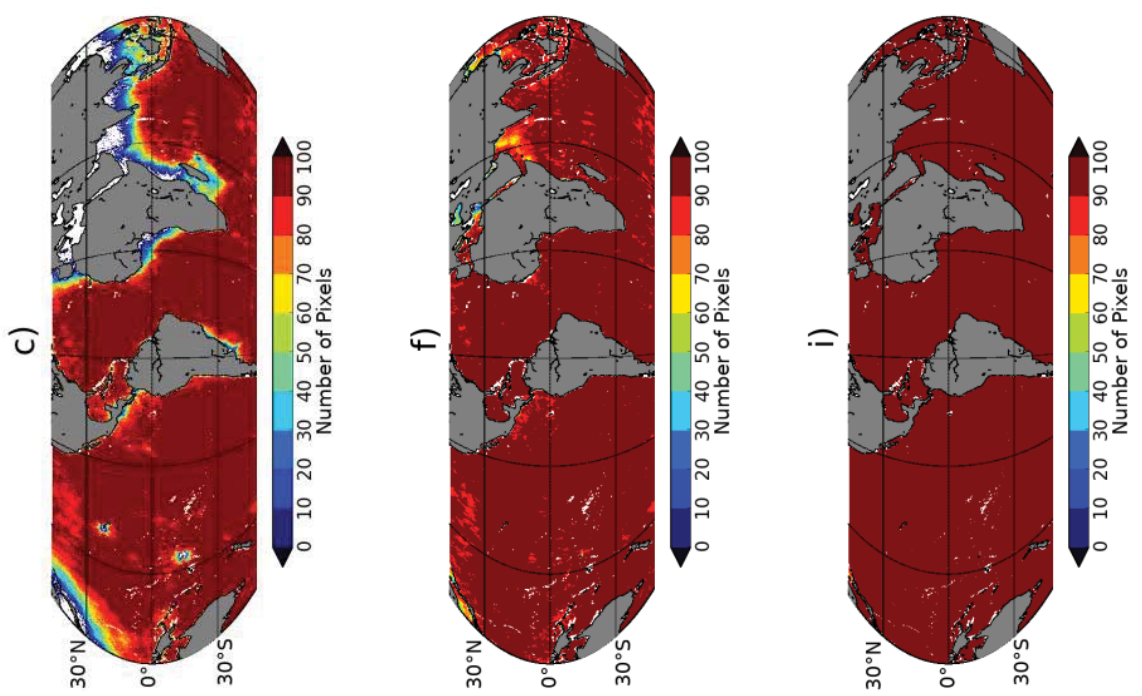

름 g

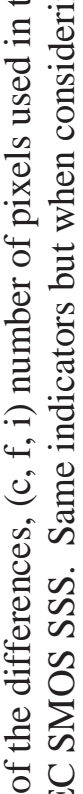
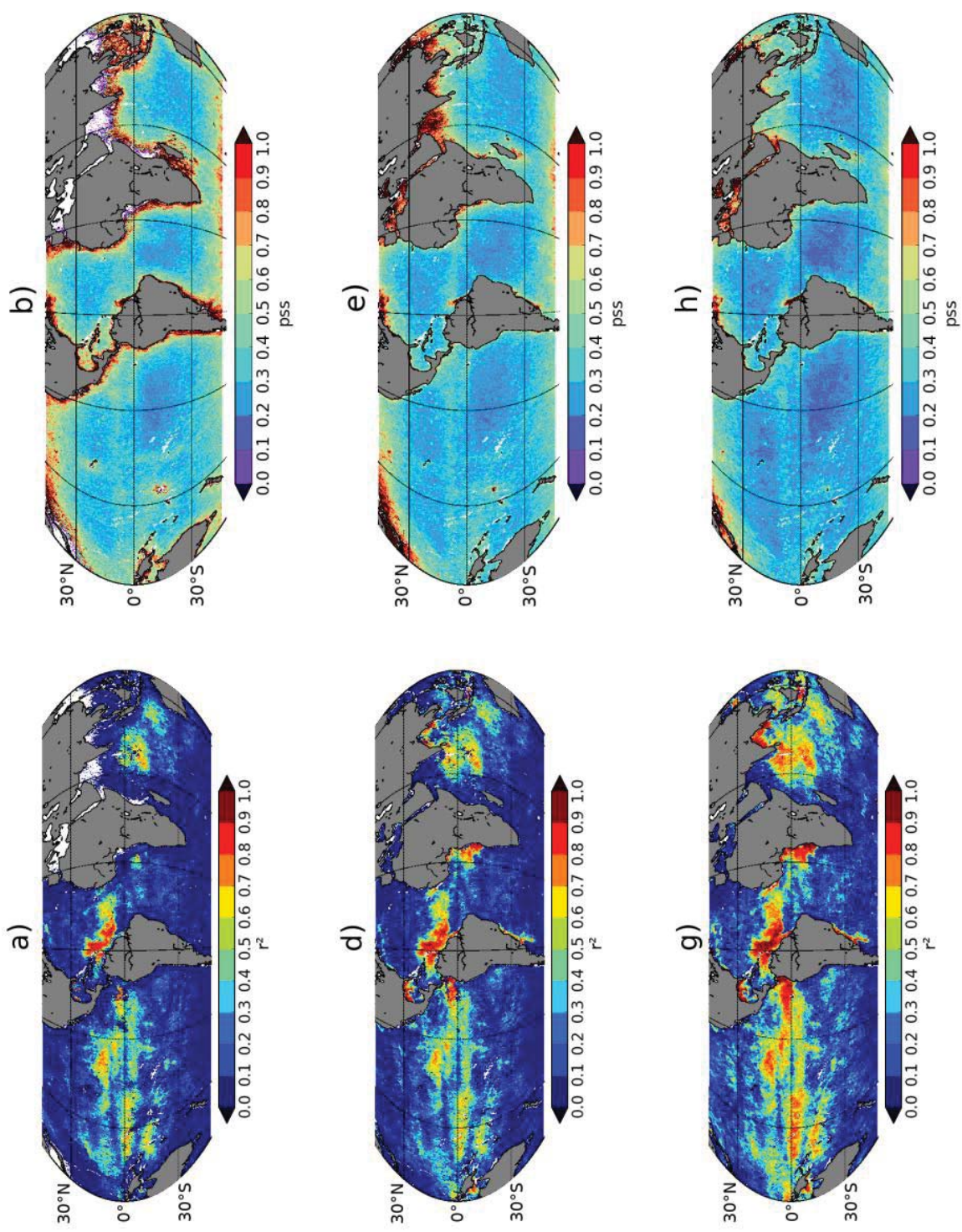

요

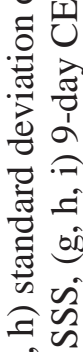

०थ

e

चै क

कo

정

ن̈

की

7

部苞

3 ज

की के

的

क

등

๗

究

넝웜

드.

은

을

ㅎํㅇำ

in

㟧

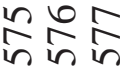


We now detail more quantitatively the comparisons between SMAP and SMOS K2016 / CEC SSS in four regions with very variable salinities (Bay of Bengal; Gulf of Mexico; Eastern Tropical Atlantic Freshwater Pools; Amazon plume), identified on Figure 5 as having a high

581 $\mathrm{r}^{2}$ after correction and already presented in the introduction. Contamination by RFI is very strong in the Bay of Bengal and in the Eastern Tropical Atlantic Freshwater Pools (see very small number of valid L3P measurements (Figure 5c)) and moderate in the two other regions. The coast geometry is very different in these 4 regions: the Bay of Bengal and Gulf of Mexico are semi-enclosed ocean areas so that land-sea contamination of an ocean pixel is expected to come from more than $290^{\circ}$ of different directions, while the other two regions are surrounded in more than $180^{\circ}$ around the points by the ocean.

As shown on the maps of Figure 1 and on the corresponding scatter plots (Figure 6, two left columns), the new SMOS CEC SSS captures fresh SSS patterns much closer to the ones in SMAP SSS and remains close to SMAP SSS in other SSS ranges. For instance, in the Bay of Bengal (Figure $1 \mathrm{a}-\mathrm{c}$ ), the comma-shaped fresh SSS around $85^{\circ} \mathrm{E}$ and $17^{\circ} \mathrm{N}$ corresponds to fresh water originating from the Ganges-Brahmaputra trapped in an eddy (Fournier et al. 2017) and the one near $15^{\circ} \mathrm{N}, 95^{\circ} \mathrm{E}$, to the Irrawady discharge. In the Gulf of Mexico (Figure $1 \mathrm{~d}$-f), the horseshoe-shaped fresh SSS coming from Texas flooding and transported by ocean currents (Fournier et al. 2016) is better captured, as well as the Eastern Tropical Atlantic Freshwater Pools (Figure $1 \mathrm{~g}-\mathrm{i}$ ) and the Amazon and Orinoco plumes (Figure $1 \mathrm{j}-\mathrm{l}$ ). The statistics of the SMOS SSS minus SMAP SSS differences are reported in Table 3. The median of the differences between SMOS and SMAP SSS and std(SMOS-SMAP) are decreased in all regions. The L1 norm estimator std1 (equal to median(abs(x-median $(\mathrm{x}))) / 0.67$, and that is less affected by the outliers than std), and $\mathrm{r}^{2}$ are clearly improved in the Bay of Bengal; the improvement is less in other regions because of the larger proportion of 
602 higher SSS values, and less stringent noise filtering at moderate SSS. For SSS less than 25pss

603 in the Amazon plume and in the Bay of Bengal, SMOS SSS remains in some cases higher 604 than SMAP SSS.

605
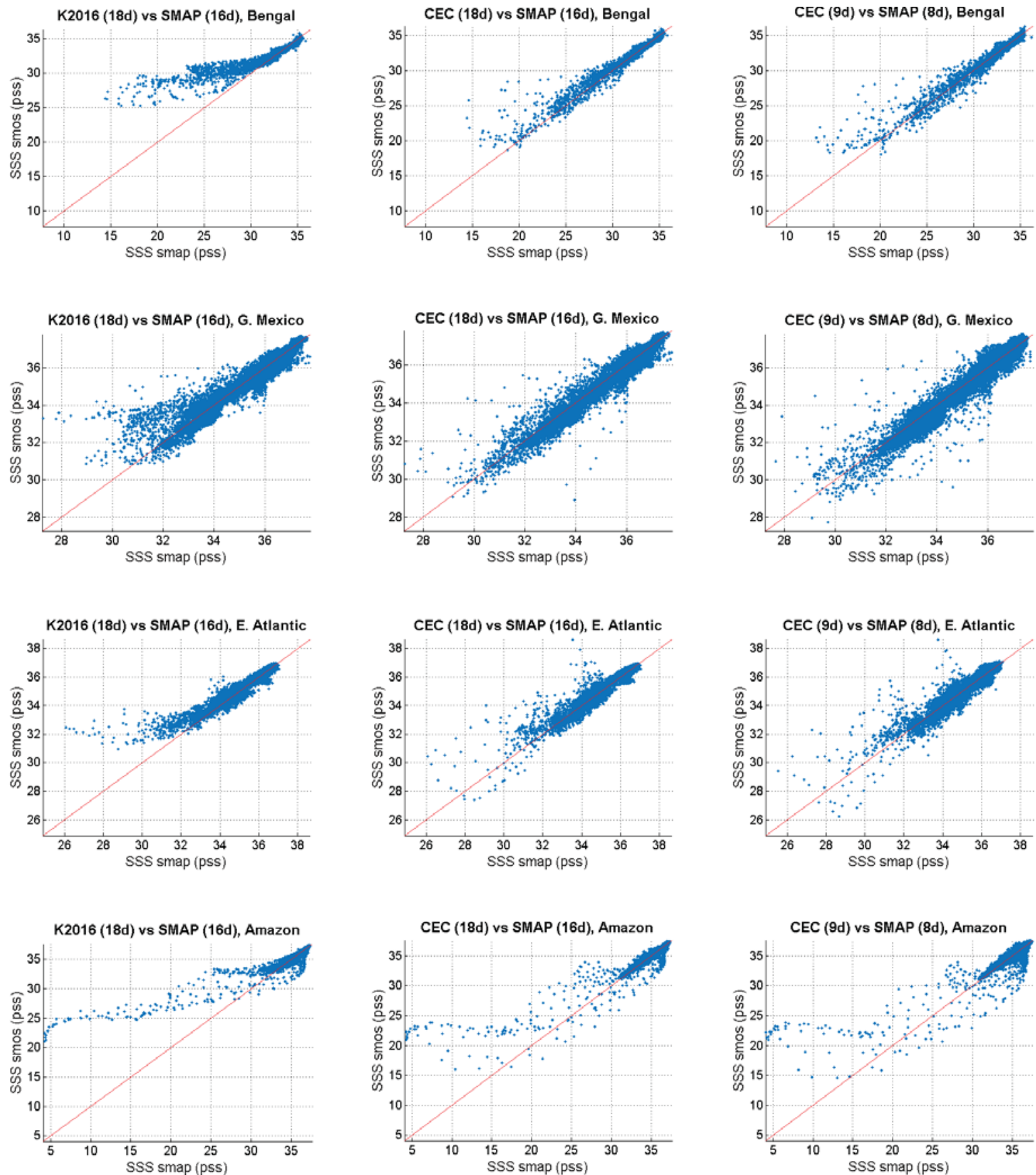
606 Figure 6 : Scatter plots of SMOS corrected fields versus SMAP SSS on the 4 regions and fresh events 607 periods illustrated on Figure 1: first line: Bay of Bengal; $2^{\text {nd }}$ line: Gulf of Mexico; 3rd line : Eastern

608 Tropical Atlantic Freshwater Pools; 4th line : Amazon plume. First column: SMOS K2016 SSS;

609 second column: SMOS 18-day CEC SSS; last column: SMOS 9-day CEC SSS.

610 Table 3: Statistics of (SMOS SSS - SMAP SSS) corresponding to scatter plots of Figure 6

\begin{tabular}{|c|c|c|c|c|c|c|c|c|c|c|c|}
\hline \multicolumn{4}{|c|}{ K2016 (18d) - SMAP (16d) } & \multicolumn{4}{|c|}{ CEC (18d) - SMAP (16d) } & \multicolumn{4}{|c|}{ CEC (9d) - SMAP (8d) } \\
\hline median & std & std1 & $r^{2}$ & median & std & std1 & $r^{2}$ & median & std & std1 & $r^{2}$ \\
\hline \multicolumn{12}{|c|}{ Bay of Bengal } \\
\hline 0.10 & 2.00 & 0.56 & 0.85 & 0.02 & 0.77 & 0.38 & 0.95 & -0.03 & 0.81 & 0.41 & 0.95 \\
\hline \multicolumn{12}{|c|}{ Gulf of Mexico } \\
\hline-0.02 & 0.50 & 0.29 & 0.90 & -0.06 & 0.39 & 0.30 & 0.94 & -0.06 & 0.45 & 0.37 & 0.93 \\
\hline \multicolumn{12}{|c|}{ Eastern Tropical Atlantic Freshwater Pools } \\
\hline 0.04 & 0.42 & 0.23 & 0.92 & 0.01 & 0.39 & 0.23 & 0.91 & 0.05 & 0.44 & 0.29 & 0.90 \\
\hline \multicolumn{12}{|c|}{ Amazon Plume } \\
\hline-0.14 & 1.00 & 0.20 & 0.83 & -0.13 & 0.82 & 0.20 & 0.85 & -0.11 & 0.87 & 0.25 & 0.80 \\
\hline
\end{tabular}

611

612 The time series of the indicators reported in Table 3 are plotted for each case study region on

613 Figure 7 to Figure 10. 'Bi-weekly' indicators confirm that during periods with large SSS

614 variability detected by SMAP (black line on top right figures) and low SSS (black line on top

615 left figures), $\mathrm{r}^{2}$ (bottom left figures) and std(SMOS-SMAP) (bottom right figures) are

616 systematically improved for CEC with respect to K2016: $\mathrm{r}^{2}$ becomes larger than 0.9 except in

617 the Amazon plume ( 0.8). This is not systematically the case during periods with low SSS

618 variability and salty SSS when sometimes K2016 performs slightly better in term of $\mathrm{r}^{2}$ and

619 std(SMOS-SMAP): this is likely because our method neglects seasonal variation of $\sigma_{\text {SSSnat. }}$

620 Nevertheless, the worse $\mathrm{r}^{2}$ obtained with CEC SSS relative to K2016 SSS correspond in

621 reality to weak degradations of the corrected SSS, given the noise in both SMOS and SMAP

622 SSS and the low SSS variability; on the contrary, the improved $\mathrm{r}^{2}$ correspond to very

623 significant improvements in the detection of fresh SSS in highly variable regions. 
624 std1 (dashed lines on bottom right figures) is on the order of 0.3 pss, which is consistent with

625 a noise on each 'bi-weekly' satellite SSS product on the order of 0.2 pss. Tang et al. 2017

626 found a standard deviation of 0.17 pss between monthly SMAP and moorings SSS over the

627 open ocean, a value comparable to the one we find with monthly-100 km SMOS-ship

628 comparisons that will be described in section 5.2.

629 The standard deviations of the SSS (SSS std (top right figures)) obtained with CEC products

630 are much closer to the SSS std of the 'weekly' SMAP products than the ones obtained with

631 the 'bi-weekly' K2016 products during highly variable periods; during periods with low

632 variability all SSS std are very close to each other. Nevertheless, except in the Gulf of

633 Mexico, SSS std are slightly larger for SMAP SSS than for CEC SSS. This possibly indicates

634 that our method still underestimates SSS natural variability in some cases. This may also be

635 due to the adjustment to the 7-year median of ISAS SSS: for instance, the fresh water along

636 the Brazil coast at $50^{\circ} \mathrm{W}-5^{\circ} \mathrm{N}$ is observed as a continuous tongue in the SMAP SSS map

637 (Figure 1l), and as a discontinuous one in the SMOS SSS maps (Figure $1 \mathrm{j}-1 \mathrm{k}$ ) which is due

638 to a discontinuity in the 7-year median of ISAS SSS (not shown). Further validation with

639 external ground truth of SMOS and SMAP SSS would be necessary to confirm the origin of

640 this discrepancy.

641 It is also instructive to consider the statistics obtained with 'weekly' products (Figure 6, right

642 column and Figure 7 to Figure 10, blue lines) as SSS during periods with large freshwater

643 discharges can be very variable at short time scales. In most cases, $\mathrm{r}^{2}$ and $\operatorname{std}(\mathrm{SMOS}-\mathrm{SMAP})$

644 obtained with 'weekly' products are slightly worse than the ones obtained with 'bi-weekly'

645 products, because the noise is higher in the 'weekly' products but it nevertheless remains

646 small relative to the natural variability. It is only in Fall, in the Bay of Bengal, when the SSS

647 std is larger than 2.5 pss, that the $\mathrm{r}^{2}$ and std(SMOS-SMAP) with the 'weekly' CEC product 
648 are comparable to the $r^{2}$ and std(SMOS-SMAP) with 'bi-weekly' CEC product, the noise

649 becoming negligible relative to the SSS natural variability. Hence, in very variable regions,

650 the 'weekly' CEC maps could improve the monitoring of fresh spatial structures varying

651 within 18 days.

652
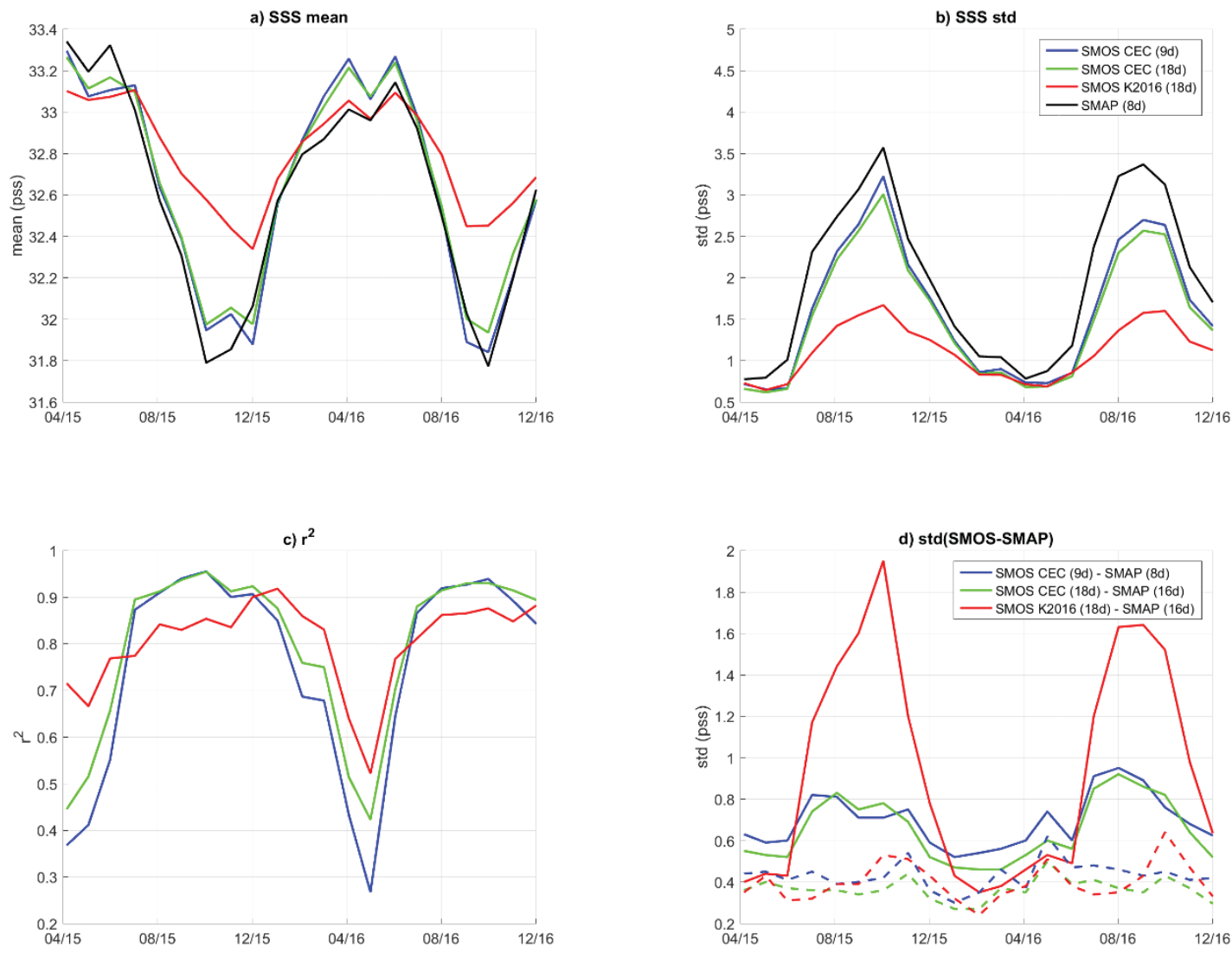

653 Figure 7: Time series of statistical parameters computed over the Bay of Bengal case study area, April 6542015 to December 2016: a) mean SSS; b) SSS standard deviation; c) square of the Pearson correlation 655 coefficient $\left(\mathrm{r}^{2}\right)$ between SMOS and SMAP SSS; d) Standard deviation of the SMOS minus SMAP 656 SSS differences (plain line) using L1 norm (dotted line). 'Weekly' SMOS CEC(blue), 'bi-weekly' 657 SMOS CEC (green), 'bi-weekly' SMOS K2016 (red), 'weekly' SMAP (black).

658 

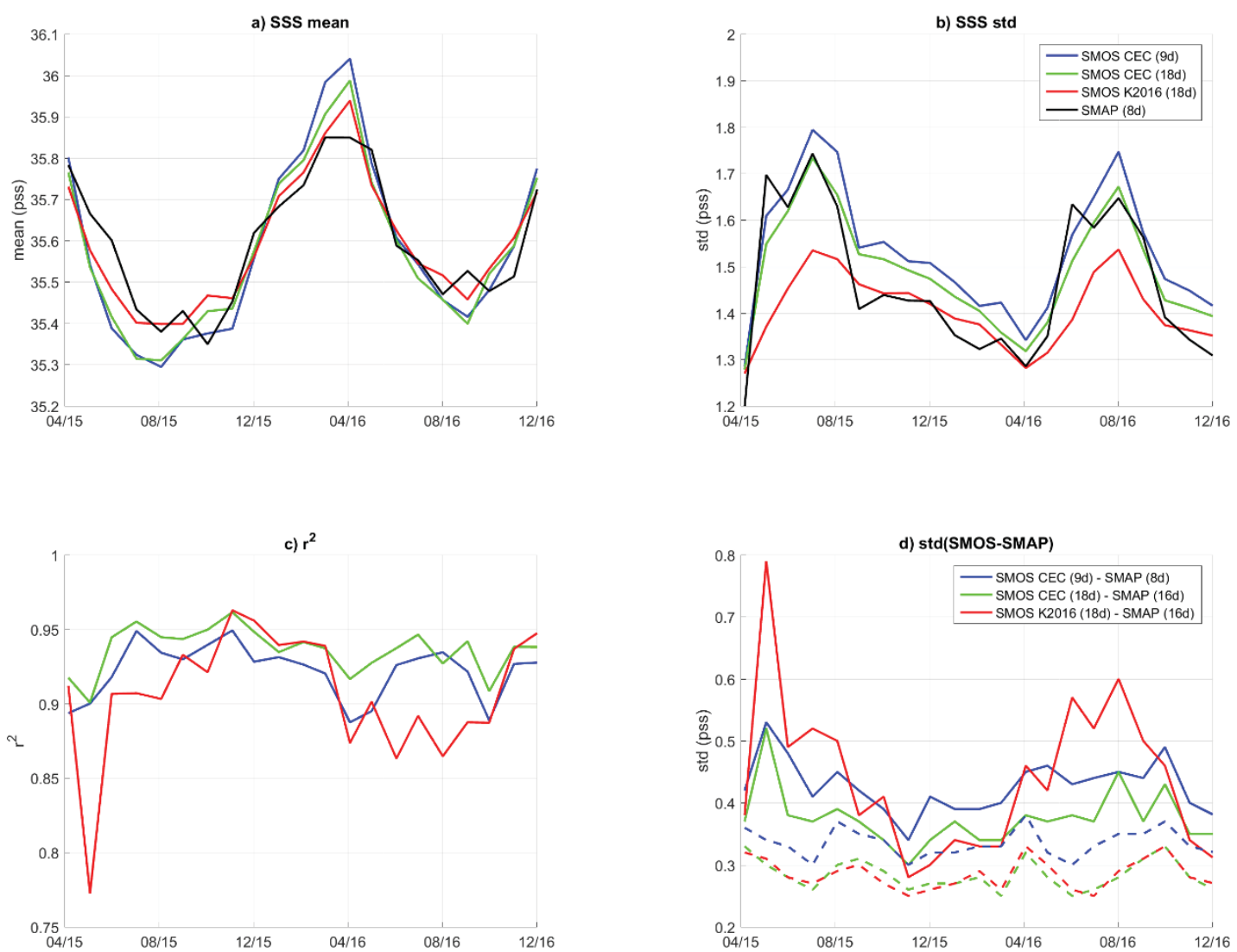

661 Figure 8: Time series of statistical parameters computed over the Gulf of Mexico case study area, 662 April 2015 to December 2016: a) mean SSS; b) SSS standard deviation; c) square of the Pearson 663 correlation coefficient $\left(\mathrm{r}^{2}\right)$ between SMOS and SMAP SSS; d) Standard deviation of the SMOS minus 664 SMAP SSS differences (plain line) using L1 norm (dotted line). 'Weekly' SMOS CEC(blue), 'bi665 weekly’ SMOS CEC (green), 'bi-weekly' SMOS K2016 (red), 'weekly’ SMAP (black). 

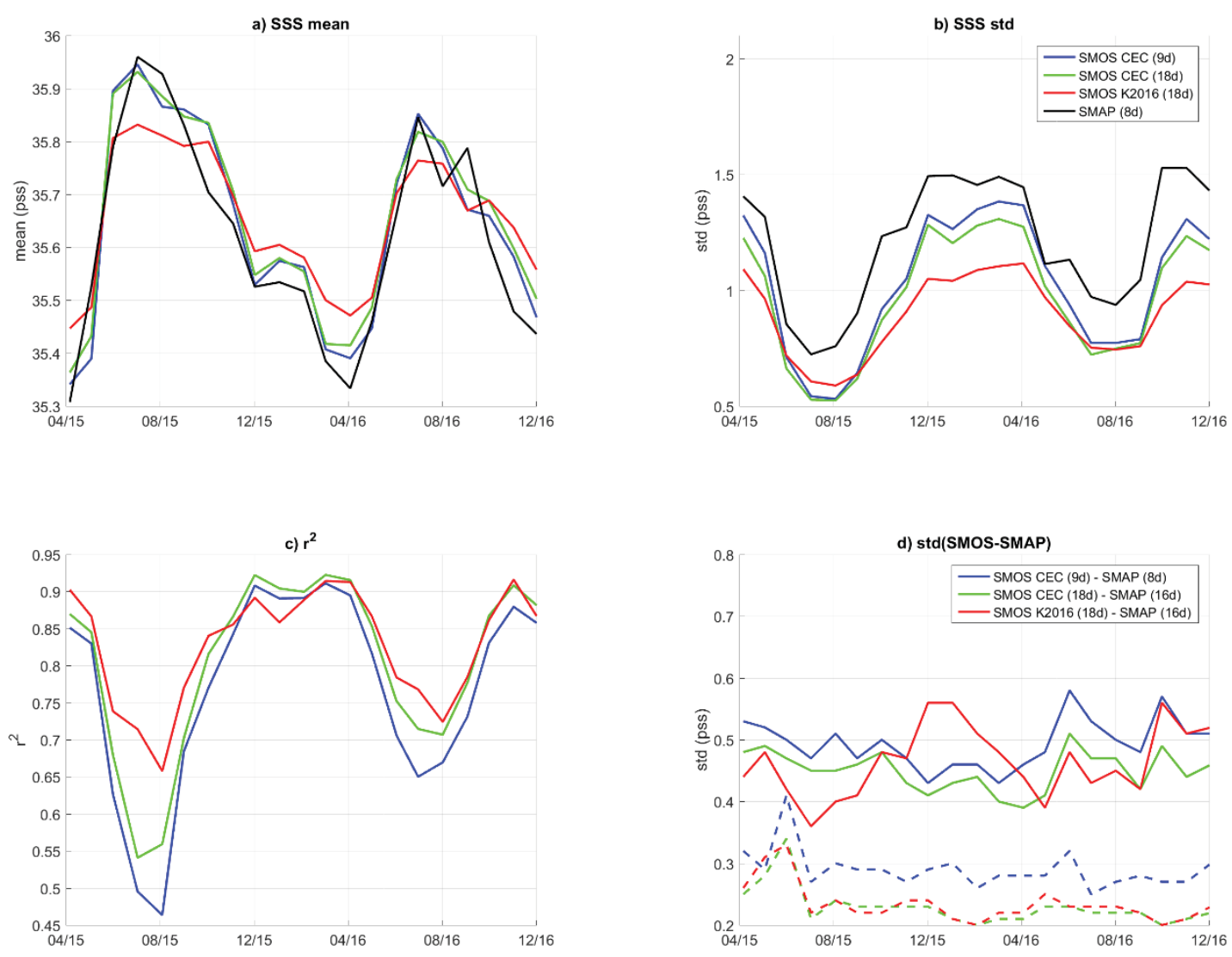

668 Figure 9: Time series of statistical parameters computed over the Eastern Tropical Atlantic Freshwater Pools case study area, April 2015 to December 2016: a) mean SSS; b) SSS standard deviation; c) square of the Pearson correlation coefficient $\left(\mathrm{r}^{2}\right)$ between SMOS and SMAP SSS; d) Standard deviation of the SMOS minus SMAP SSS differences (plain line) using L1 norm (dotted line). 'Weekly' SMOS CEC(blue), 'bi-weekly' SMOS CEC (green), 'bi-weekly' SMOS K2016 (red), 673 'weekly' SMAP (black).
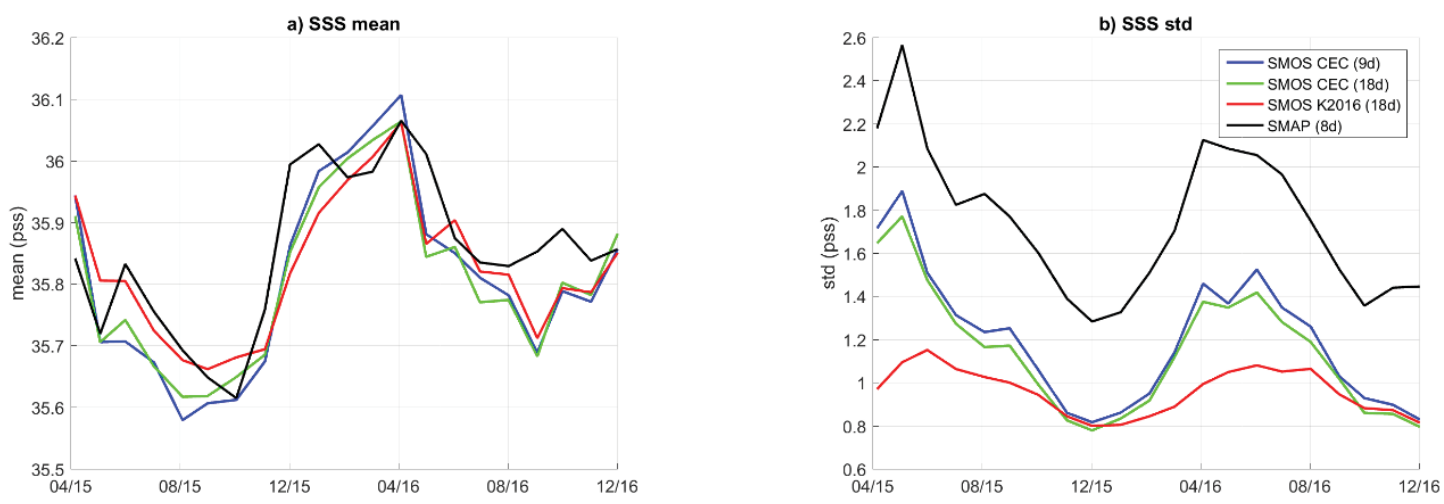

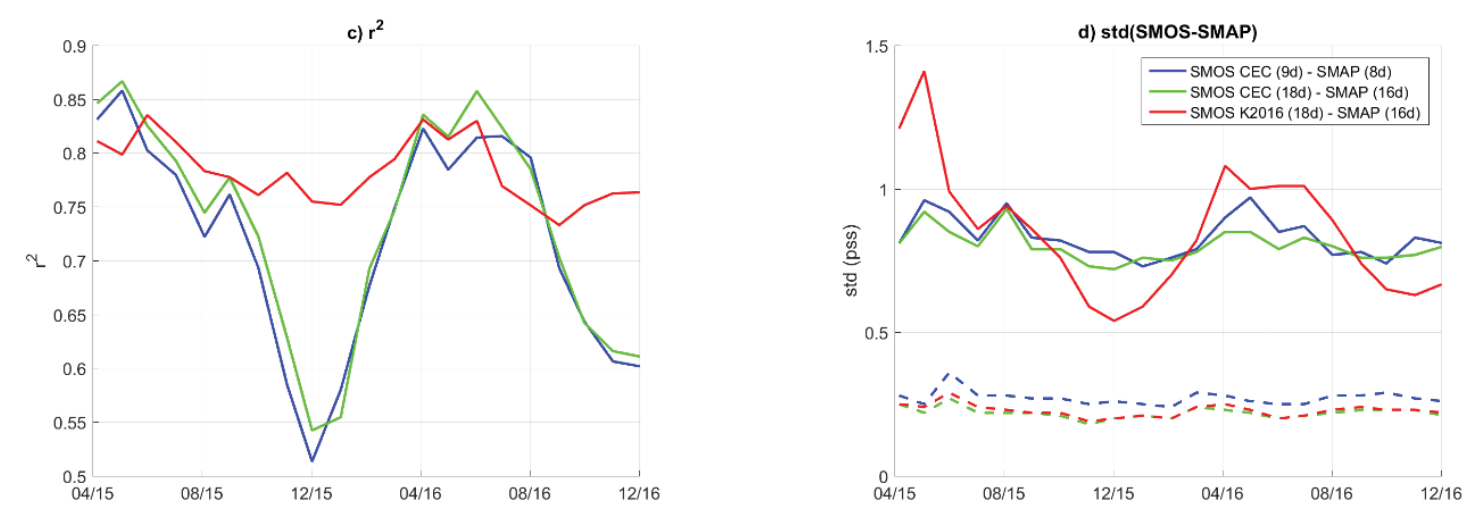

675 Figure 10: Time series of statistical parameters over the Amazon plume case study area, April 2015 to

676 December 2016: a) mean SSS; b) SSS standard deviation; c) square of the Pearson correlation

677 coefficient $\left(\mathrm{r}^{2}\right)$ between SMOS and SMAP SSS; d) Standard deviation of the SMOS minus SMAP

678 SSS differences (plain line) using L1 norm (dotted line). 'Weekly' SMOS CEC(blue), 'bi-weekly'

679 SMOS CEC (green), 'bi-weekly’ SMOS K2016 (red), ‘weekly’ SMAP (black).

680

\subsection{Comparison to ship SSS}

682

Merchant ship transects are used to get ground-truth measurements at various distances from

683

the coast. With respect to SMAP SSS, ship SSS is less uncertain but its spatio-temporal

684

sampling and resolution is very different from SMOS SSS.

685

In a first step, we consider the scales of SSS variability captured by the various SMOS SSS

686

versions and by the ship SSS far from coast. We focus on the subtropical region $\left(50^{\circ} \mathrm{W}\right.$ -

687

$20^{\circ} \mathrm{W} ; 15^{\circ} \mathrm{N}-40^{\circ} \mathrm{N}$ ) of the north Atlantic in 2013 . This region is chosen because it is very

688

well covered by regular ship tracks spaced by approximately one month, it is strongly

689

impacted by the seasonally-varying latitudinal biases, it is characterized by mesoscale

690 variability that is not resolved by the ISAS analysis (Kolodziejczyk et al. 2015; Sommer et al.

691 2015), and it is not used for choosing the reference dwell lines of the seasonal latitudinal

692

correction. We analyze below the density spectra (Figure 11, top) and the squared coherence

693 (Figure 11, bottom) of ISAS, of 10-day L3P and L3Q, of 18-day CEC with ship SSS. Our

694 analysis focuses on wavelengths between $1400 \mathrm{~km}$ and $150 \mathrm{~km}$, in order to minimize the 
influence of the limited length of the selected ship tracks (about $2800 \mathrm{~km}$ ) and of scales

696

697

698

699

700

701

702

703

704

705

706

707

708

709

710

711

712

713

714

715

716

717

718

resolved by SMOS (50 km). We recall here that coherence quantifies the correlation between two quantities for a given wavenumber band. While at $1400 \mathrm{~km}$ wavelength ISAS and ship SSS are very coherent, due to the subsampling of Argo measurements (1 profile per 10 days per $\left.3^{\circ} \times 3^{\circ}\right)$ and to the horizontal scales of the optimal interpolation $(\sim 300 \mathrm{~km})$, the ISAS spectrum (Figure 11, top, green line) dramatically drops as well as its squared coherence (Figure 11, bottom) for shorter wavelengths.

Whatever the wavelength, the density spectra (Figure 11 top) of the 18-day CEC SSS is closer to the one of the ship SSS than the 10-day L3P and L3Q. The density spectrum of the 9-day CEC SSS is very similar to the one of the 10-day L3Q for spatial wavelengths between 150 and $330 \mathrm{~km}$. For longer wavelengths, the density spectrum of 9-day CEC SSS is intermediate between the 18-day CEC and the 10-day L3Q, indicating that at large scale, where the temporal variability between 9 days and 18 days is expected to be small, the different filtering and gaussian mapping applied to CEC products is more effective at reducing the SMOS SSS noise than the min/max filtering and bin average mapping applied to L3Q products. Up to $150 \mathrm{~km}$, the density spectra of the 18-day CEC and ship SSS are in remarkable agreement. This is in fact quite surprising because the MIRAS and TSG instrumental noises are not expected to lead to the same SSS errors and because the temporal sampling of SMOS (about 8 passes over 18 days) and of ship ( one transect per month) are very different. Given the expected noise in level 2 SMOS retrieved SSS (0.6 pss), the median filtering over nearest neighbor pixels at $25 \mathrm{~km}$ distance in the SMOS CEC product, and the SMOS temporal sampling, the noise on the 18-day CEC SSS is expected to be on the order of 0.15 pss. Noise on individual ship SSS is estimated to be less, on the order of 0.08 pss (Alory et al. 2015) but the temporal sampling is worse. Hence, the similarity in the two density 
719 spectra suggests that the SSS error due to instrumental noise that is larger in SMOS than in

720 ship SSS, is compensated, over 18 days, by the better temporal sampling in SMOS than in the

721 ship data.

722 The squared coherence (Figure 11 bottom) of the 18-day CEC SSS is almost at the same level

723 (above 0.7) as the squared coherence of ISAS SSS at a $1400 \mathrm{~km}$ wavelength, and is always at

724 a higher and significant level for wavelengths up to $300 \mathrm{~km}$. The 18-day CEC squared

725 coherence decreases with decreasing spatial wavelengths. This can be due to instrumental

726 noise, to the different temporal sampling of SMOS and ship and to spatially moving

727 structures within 18 days. The 18-day CEC squared coherence becomes not significant at

$72895 \%$ for wavelengths smaller than $300 \mathrm{~km}$. Considering that at least 3 samples are necessary

729 to resolve a $300 \mathrm{~km}$ wavelength signal, this result indicates that 18-day CEC and ship SSS

730 capture similar scales of variability up to about $100 \mathrm{~km}$. This is rather consistent with the

731 spatial integration of SMOS measurement $(50 \mathrm{~km})$ in addition to the median filtering over

732 nearest neighbor pixels at $25 \mathrm{~km}$ applied on CEC products.

733 The level of coherence is much less both with the 10-day L3P and L3Q products, due to a

734 lower signal to noise ratio. 

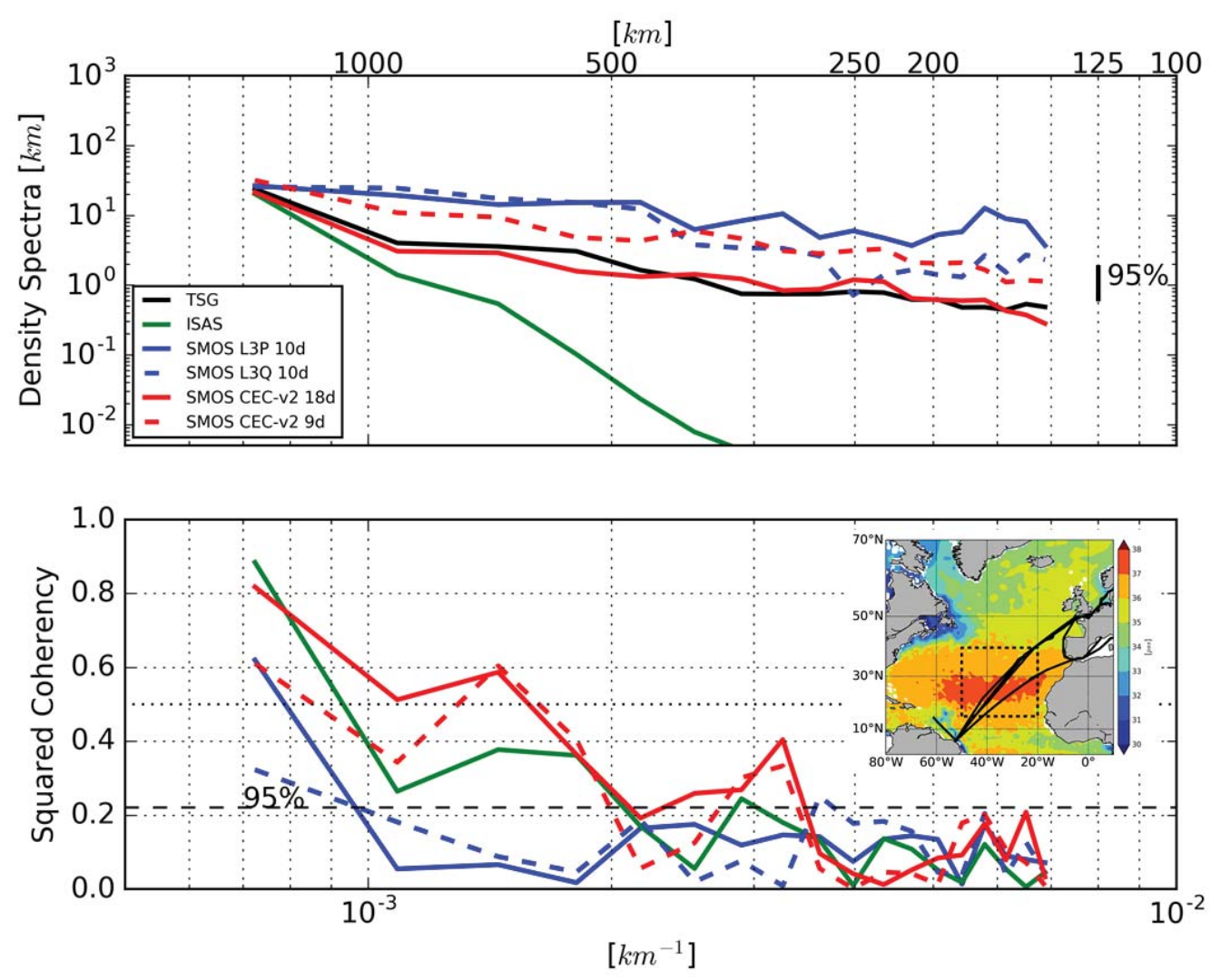

Figure 11 : Top: Density spectra; Bottom: Coherence between ship SSS and SMOS or ISAS SSS. The spatial frequency (1/wavelength $(\mathrm{km}))$ is indicated below the bottom plot, whereas the corresponding wavelengths $(\mathrm{km})$ are indicated above the top plot. Vertical dashed lines correspond to spatial frequencies regularly spaced in logarithmic coordinates. Northern subtropical Atlantic (see box on the color map) in 2013. Ship SSS measured on regular merchant ships transects (14 regular transects in 2013) (black), ISAS SSS (green), 10-day SMOS L3P (blue line), 10-day SMOS L3Q (dashed blue line), 18-day SMOS CEC (red line), 9-day SMOS CEC (red dashed line).

We will now investigate global statistics for the difference between SMOS and ship SSS.

745 Consistent with the weak coherence observed between the ship SSS and the 10-day L3P and monthly L3P, L3Q and 18-day CEC fields. Ships provide within a few hours numerous measurements within a satellite pixel. In the following, the SSS variability sampled by each ship and by SMOS is smoothed over $+/-50 \mathrm{~km}$. This smoothing cannot be identical for the 
two platforms because of their different spatio-temporal sampling. SMOS observes a surface

752

753

754

755

756

757

758

759

760

761

762

763

764

765

766

767

768

769

770

771

772

773

774

(two dimensions) whereas ship measurements are taken along a route (one dimension).

However, this method is expected to reduce the misfit between in situ and SMOS

observations coming from the spatial subsampling of SSS variability within a satellite pixel

by point measurements (Boutin et al. 2016). Mean differences and standard deviation of the differences between SMOS SSS and ship SSS, named Std(SMOS-Ship) in the following, are shown in Figure 12, as a function of the distance from the coast. Two sets of comparisons are presented, involving either only SMOS pixels common to L3P fields (i.e. the ones the less affected by RFI pollution) (Figure 12, left) or all valid pixels for each product (Figure 12, right). The number of valid pixels is increased by nearly a factor 2 when approaching the coast with L3Q and CEC fields with respect to L3P fields (Figure 12, bottom right). The mean differences (Figure 12, top) obtained with monthly L3P are less than -0.5 pss up to 600 $\mathrm{km}$ from the coast. The mean differences with CEC fields are systematically less than 0.05 pss (in absolute value), further than $100 \mathrm{~km}$ from the coast, a very clear improvement with respect to L3P. Similar improvement is observed with monthly L3Q when considering only pixels common to L3P (Figure 12, left); the mean differences are, however, slightly more negative when considering all valid pixels, indicating that the filtering is more efficient at removing SSS outliers in CEC than in L3Q processing. At less than $100 \mathrm{~km}$ from the coast, the mean difference with CEC product reaches 0.15 pss, a value close to the mean difference between ISAS and ship SSS, consistent with local overestimate of the long term SSS mean by ISAS, as suggested by SMOS and SMAP SSS comparisons in the Amazon plume along the coast (Figure 1 and section 5.1). However, the scatter plot (not shown) between CEC and ship SSS in the region of the Amazon plume is very scattered at low SSS and it was not possible to identify a systematic bias. 
775

776

777

778

779

780

781

782

783

784

785

786

787

788

789

790

791

792

793

794

795

796

797

Std(SMOS-Ship) is clearly improved whatever the distance to the coast. Further than 1000 $\mathrm{km}$ from the coast, it is equal to 0.20 pss with CEC, 0.21 pss with L3Q while it is 0.24 pss with L3P. It increases when approaching the coast: in the 100-200 km class and when considering all valid pixels, it equals to 0.64 pss with CEC, 0.69 pss with L3Q, 0.78 pss with L3P. When approaching the coast, the ship SSS variability is increased too (black lines on Figure 12, middle right) and it is likely that part of the Std(SMOS-Ship) induced by the different temporal sampling of SMOS and ships increases when approaching the coast. Consequently, while Std(SMOS-Ship) is increased by a factor 3 between 100-200 km and further than $1000 \mathrm{~km}$ from the coast, the signal to noise ratio is increased by only a factor 1.5 between these two classes. Similarly, Std(SMOS-Ship) and ship SSS std are lower when considering only L3P pixels than when considering all valid pixels, so that the signal to noise ratio in both cases remains similar. When considering all valid pixels (Figure 12, middle right), the std difference obtained with ISAS remains slightly less than the ones obtained with CEC and L3Q SSS in all the classes considered except for the range from 500 to $900 \mathrm{~km}$ (Figure 12, middle right). On the contrary, when considering only pixels common to L3P, (Figure 12, middle left), CEC SSS better captures SSS variability than ISAS in all the classes up to $900 \mathrm{~km}$ from a coast. Two typical ship comparisons illustrate these features. On Figure 13 (left), a transect in the South Pacific is quite well sampled by L3P, except between the equator and $4^{\circ} \mathrm{N}$ where the L3Q and CEC SSS is closer to ship SSS. ISAS SSS appears to be smoother than SMOS SSS, as expected from the optimal interpolation. On Figure 13 (right) a ship transect crosses the North Atlantic Ocean in September 2013, a period of moderate RFI. The L3P SSS is very discontinuous due to RFI disturbances in the north and to land-sea contamination south of the equator. The L3Q and CEC SSS are more numerous and closer to 

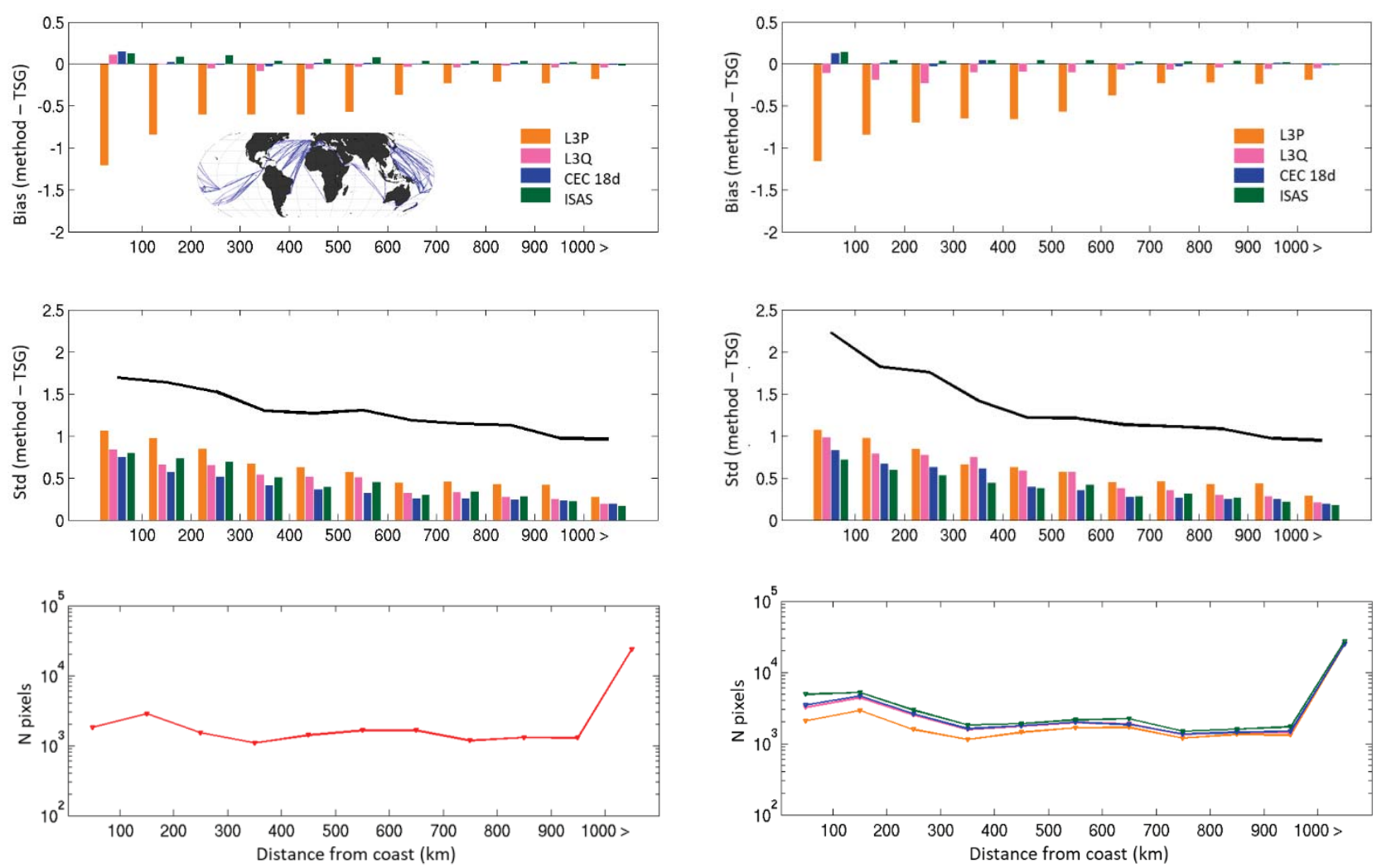

800 Figure 12 : Statistics of ship comparisons (May 2010-August 2016) binned as a function of the

801 distance from the nearest coast: top) mean difference; middle) standard deviation of the differences;

802 the black line indicates the standard deviation of ship SSS in each class; bottom) number of pixels

803 used in the comparisons. Left: considering only the SMOS pixels common to all versions; right:

804 considering all pixels available in each version. Ship and SMOS SSS are integrated over $100 \mathrm{~km}$.

805 Orange: monthly SMOS L3P ; pink : monthly SMOS L3Q; light blue : 18-day SMOS CEC; green :

806 ISAS. 

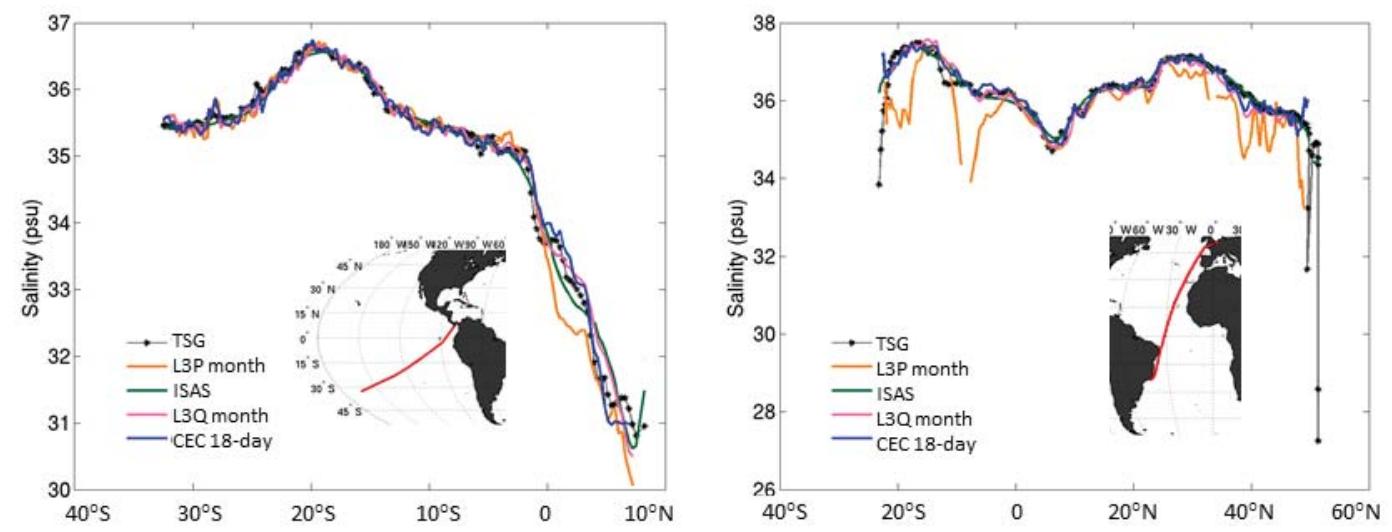

808

809 Figure 13 : Examples of comparisons between ship SSS (black stars line) and SMOS SSS: orange:

810 non corrected (L3P), purple: monthly L3Q corrected, light blue :18-day CEC corrected; green : ISAS.

811 Left) from 2014-08-21 to 2014-09-03, Matisse ship. Right) from 2013-08-21 to 2013-09-03, Santa

812 Cruz ship. All SSS products have been smoothed over $+/-50 \mathrm{~km}$.

813

\section{Discussion and Perspectives}

815 Retrieving accurate SSS from SMOS measurements in the vicinity of continents is very

816 challenging. The land-sea brightness temperature contrasts induce a contamination of the

817 retrieved SSS signal, up to about $1000 \mathrm{~km}$ from the coast. This contamination is very variable

818 across the SMOS swath. The origin of this pollution is very complex. It is likely related to an

819 imprecise characterization of the 69 individual antenna patterns constituting the SMOS

820 synthetic antenna, preventing a reliable theoretical modelling of the correction in the current

821 SMOS image reconstruction process. The land-sea contamination has thus to be mitigated

822 empirically. When doing so, the main difficulty is to distinguish the SMOS signal resulting

823 from natural SSS variability from ones contaminated by RFI, whose sources are often located

824 near coasts. To make matters worse, the typical RFI signature yields low SSS, and the largest

825 SSS natural variability often occurs in low SSS regions, e.g. from river plumes or high rain

826 regime. The K2016 methodology developed for correcting SSS affected by land-sea

827 contamination was very efficient in many areas, but not in those characterized by strong 
natural variability, as it implicitly assumed that natural SSS variability was negligible relative to SMOS SSS noise. The revised correction methodology presented in this paper includes information on the amplitude of natural SSS variability inferred from SMOS measurements.

831 We further add a seasonally- and latitudinally-dependent bias correction.

832 The SMOS corrected SSS is much more consistent to the independent SMAP SSS than 833 K2016, both in terms of SSS patterns and amplitude (Table 3). The SMOS SSS is, however, 834 slightly noisier than SMAP: in the open ocean (Pacific ITCZ region), Supply et al. 2017 835 found an error of 0.6 pss on L2 SMOS SSS and of 0.5 pss on L2 SMAP SSS. This difference 836 is explained by the radiometric accuracy of the respective instruments and by the SMAP 837 flight hardware that allows efficient detection and filtering of most RFI (Mohammed et al. 2016) unlike SMOS. Nevertheless, both satellite missions record very similar SSS variability at weekly time scale that is not resolved by mapped Argo data (Figure 14). On average over $47^{\circ} \mathrm{N}-47^{\circ} \mathrm{S}$, the standard deviation of the difference between SMOS CEC and ISAS SSS

841 (Figure 14a) is 0.33 pss while the standard deviation of the difference between SMAP and

842 ISAS SSS (Figure 14b) is 0.31 pss. The geographical distribution of this variability is very 843 consistent with the small-scale variability of SSS observed by ship measurements (see Figure 8446 of Boutin et al. 2016) with minima in the subtropics and maxima in coastal areas, in the

845 vicinity of river plumes or in regions characterized by strong mesoscale fronts, such as the 846 Gulf Stream.
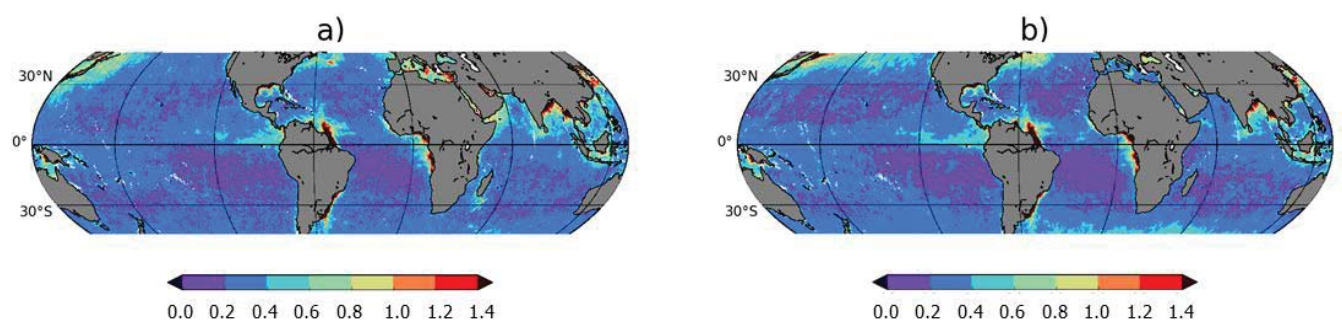
Figure 14 : Standard deviation of 'weekly' satellite SSS minus ISAS SSS between $47^{\circ} \mathrm{N}$ and $47^{\circ} \mathrm{S}$, over the year 2016. a) SMOS CEC, b) SMAP CAP.

850

851 The only quantitative external information entered in the correction algorithm is the 7-year

852 median of ISAS SSS that fixes the absolute calibration of the SMOS SSS in each pixel but 853 does not influence its variability. In seasonally-varying latitudinal biases correction, ISAS

854 SSS serves only in a qualitative way for choosing the SMOS cross-swath locations used as 855 reference. The implemented correction removes most of the systematic errors and brings clear improvement when compared with in situ ground truths measurement or with SMAP SSS. Nevertheless, some refinements could still be envisioned. The absolute calibration based on ISAS median SSS leads to some inaccuracies in very near coastal pixels. This issue could probably be improved in the future by analyzing to what extent the absolute calibration is sensitive to the time period under consideration for computing the median and by merging information coming from ISAS SSS with other SSS fields. A further step could be taken by merging SMOS and SMAP information in order to build a level 4 product taking advantage of synoptic spatio-temporal coverage of satellite data for monitoring SSS variability and using in situ SSS for the absolute calibration of SSS fields. Future studies should also pay more attention to the bias seasonal and interannual variability as a function of sun activity and of land Tb variability which have been neglected in our study.

867 Our method corrects SMOS SSS retrieved with a Bayesian approach at level 2, as described 868 in Zine et al. 2008 and as implemented in ESA and CATDS operational processors. Such a 869 retrieval method takes advantage of the expected consistency between the various Tbs measured at various incidence angles at a given distance across the swath and takes the

871 radiometric accuracy of each Tb into account. The land-sea contamination is expected to add 872 variability and biases on the SMOS Tbs at a given distance across the swath, so that the 
873 quality of the Bayesian retrieval is downgraded. In order to cope with this caveat, a

874 systematic correction at Tb level has been implemented in ESA L2 OS processor v662,

875 before the retrieval of SSS. The biases in the vicinity of land and the standard deviation of the

876 difference with respect to ISAS are much reduced (Spurgeon and SMOS-Ocean Expert

877 Support Laboratories, 2017), but flagged SSS (poor quality retrieval) remain in many coastal

878 areas. Thus, the accuracy of the retrieved SSS is in general not as good as the one obtained

879 with our correction at the SSS level (Level-3). The better performance of our methodology is

880 likely due to the fact that we account for SSS variability. Given all the non-SSS geophysical

881 effects affecting Tbs (roughness effect, galactic noise etc...), it is very difficult to account for

882 SSS variability when dealing with Tbs measured at different angles within the field of view.

883 Nevertheless, future work should explore a two-step correction, first performed at Tb level to 884 improve the Bayesian L2 retrieval and second performed at SSS level.

885 An alternative debiasing method from a non-Bayesian approach has also been proposed by

886 Olmedo et al (2017). Contrary to our approach, Olmedo et al. (2017) retrieve SSS from single 887 angular Tb measurements, they filter SSS outliers using statistical indicators of the 3-year 888 SSS histogram per incidence angle classes. They adjust the absolute value of SMOS SSS by 889 adding the World Ocean Atlas climatology. An analysis (not shown) of the 9-day De-biased 890 non-Bayesian SMOS SSS fields available from the Barcelona Expertise Center which have

891 been obtained with an objective analysis in the regions and periods shown in Figure 1

892 indicates that the striking fresh features are captured at a similar level as what was obtained 893 with K2016 methodology, consistent with the fact that the statistical indicators used to filter 894 outliers do not depend on the SSS natural variability.

895 While SMAP SSS is expected to be much less affected by RFI, some disturbances remain in 896 some regions (Mohammed et al. 2016) and the calibration of SMAP data is also challenging 
897 (Fore et al. 2016b, Meissner and Wentz, 2016). Hence, when dealing with a local scientific 898 study, dedicated comparisons with in situ ground truth are highly recommended in order to 899 precisely estimate the validity of satellite SSS in a given region and period with respect to the

900 natural variability that is considered. This should be facilitated in the future with the

901 development of the SMOS PIlot Mission Exploitation Platform (PI-MEP).

902 The CATDS/CPDC L3Q SSS is currently limited to $47^{\circ} \mathrm{S}-47^{\circ} \mathrm{N}$ as we could not define

903 unbiased SMOS reference dwell lines poleward of this latitude. This is likely because of

904 imperfect correction for surface roughness and ice contamination which can extend up to

$9051000 \mathrm{~km}$ from the ice edge and which is much more difficult to mitigate than land-sea

906 contamination as the ice edge is moving. Future studies should focus at correcting the ice

907 contamination and improving roughness correction. In addition, in regions contaminated with

908 highly variable RFI over the 7-year period, such as the northernmost parts of the Atlantic and

909 Pacific Oceans, the land-sea contamination correction becomes very tricky. In our study, RFI

910 affected Tbs are filtered out using a three-sigma filtering applied on SMOS Tbs before

911 retrieving SSS and using a Chi filtering applied on L2 SSS. Future studies should look at

912 improving this filtering.

913

\section{Acknowledgements}

914 We thank Stéphane Tarot for his very reactive support at CATDS CPDC. This work was

915 supported by CNES-CATDS and CNES-TOSCA SMOS-Ocean projects. SMOS CATDS

916 CEC data (also named L3_DEBIAS_LOCEAN_v2) have been produced by LOCEAN/IPSL

917 (UMR CNRS/UPMC/IRD/MNHN) laboratory and ACRI-st company. CATDS-CPDC data

918 (freely available at the CATDS) were operated for the "Centre National d'Etudes Spatiales"

919 (CNES, France) by IFREMER (Brest, France). Both products are available at www.catds.fr. 
SMAP CAP SSS is produced at Jet Propulsion Laboratory. It is available on ftp://podaac-

921

922

923

924

925

926

927

928

929

930

931

932

933

934

935

936

937

938

939

940

941

942

943

944

945

946

947

948

949

950

ftp.jpl.nasa.gov/allData/smap/. ISAS 13 analysis fields are made freely available by

Laboratoire d'Océanographie Physique et Spatiale (LOPS) (http://www.umr-lops.fr/SNO-

Argo/Products/ISAS-T-S-fields). Near real time ISAS analysis are produced by the Coriolis

data center and are freely available via CMEMS web site CMEMS-Copernicus Services:

http://marine.copernicus.eu/. SSS data derived from thermosalinograph instruments installed onboard voluntary observing ships were collected, validated, archived, and made freely available by the French Sea Surface Salinity Observation Service (http://www.legos.obsmip.fr/observations/sss/). We thank anonymous reviewers for their comments which helped to improve the manuscript.

\section{REFERENCES}

Akhil, V. P., M. Lengaigne, F. Durand, J. Vialard, A. V. S. Chaitanya, M. G. Keerthi, V. V. Gopalakrishna, J. Boutin, and C. de Boyer Montégut (2016), Assessment of seasonal and year-to-year surface salinity signals retrieved from SMOS and Aquarius missions in the Bay of Bengal, International Journal of Remote Sensing, 37(5), 1089-1114, doi:10.1080/01431161.2016.1145362.

Alory G., T. Delcroix, P. Téchiné, D. Diverrès, D. Varillon, S. Cravatte, Y. Gouriou, J. Grelet, S. Jacquin, E. Kestenare, C. Maes, R. Morrow, J. Perrier, G. Reverdin, and F. Roubaud (2015), The French contribution to the Voluntary Observing Ships network of Sea Surface Salinity. Deep Sea Res., 105, 1-18, doi:10.1016/j.DSR.2015.08.005.

E. Anterrieu, M. Suess, F. Cabot, P. Spurgeon and A. Khazâal (2015), An Additive Mask Correction Approach for Reducing the Systematic Floor Error in Imaging Radiometry by Aperture Synthesis," in IEEE Geoscience and Remote Sensing Letters, vol. 12, no. 7, pp. 1441-1445, doi: 10.1109/LGRS.2015.2406912.

Arias, M. and SMOS-Ocean Expert Support Laboratories, 2016, L2OS v622 Reprocessing Report, SO-RP-ARG-GS-0100 Issue:2, 29 February 2016, available on https://smos.argans.co.uk/docs/technotes/SO-RP-ARG-GS0100_L2OS_Reprocessing_Report_v2.0_160229.pdf.

Boutin J., Vergely J.L., Marchand S. (2017). SMOS SSS L3 debias v2 maps generated by CATDS CEC LOCEAN. http://doi.org/10.17882/52804. 
951

952

953

954

955

956

957

958

959

960

961

962

963

964

965

966

967

968

969

970

971

972

973

974

975

976

977

978

979

980

981

982

983

984

985

986

987

988

989

990

Bretherton, F.P., R.E. Davis and C.B. Fandry (1976) Technique for Objective Analysis and Design of Oceanographic Experiments Applied to Mode-73. Deep-Sea Research. 23:559-582.

Brodzik, M. J., B. Billingsley, T. Haran, B. Raup, M. H. Savoie (2012) EASE-Grid 2.0: Incremental but Significant Improvements for Earth-Gridded Data Sets. ISPRS International Journal of Geo-Information, 1(1):32-45, doi:10.3390/ijgi1010032.

CATDS (2017a). CATDS-PDC L3OS 2P - Daily valid ocean salinity values product from SMOS satellite. CATDS (CNES, IFREMER, LOCEAN). http://doi.org/10.12770/77edd3084296-4774-b6f3-5b38301cee18.

CATDS (2017b). CATDS-PDC L3OS 3P mixed - Average 10 days \& monthly salinity field product from SMOS satellite (mixed orbits). CATDS (CNES, IFREMER, LOCEAN). http://doi.org/10.12770/75ccd428-74b5-45db-879e-37ab98fa28a1

CATDS (2017c). CATDS-PDC L3OS 3Q mixed - Debiased average 10 days \& monthly salinity field product from SMOS satellite (mixed orbits). CATDS (CNES, IFREMER, LOCEAN, ACRI). http://doi.org/10.12770/0f02fc28-cb86-4c44-89f3-ee7df6177e7b

Chaittanya, A.V.S., M. Lengaigne, J. Vialard, V.V. Gopalakrishna, F. Durand, Ch. Krantikumar, V. Suneel, F. Papa and M. Ravichandran (2014), Fishermen-operated salinity measurements reveal a "river in the sea" flowing along the east coast of India, Bull. Am. Met. Soc., 95, 1897-1908.

Delcroix, T., M. McPhaden, A. Dessier, and Y. Gouriou (2005), Time and space scales for sea surface salinity in the tropical oceans. Deep Sea. Res., 52/5, 787-813, doi:10.1016/j.dsr.2004.11.012.

Durack, P. J., Wijffels, S. E., and Matear, R. J. (2012), Ocean salinities reveal strong global water cycle intensification during 1950 to 2000. Science, 336(6080), 455-458.

Font, J., A. Camps, A. Borges, M. Martin-Neira, J. Boutin, N. Reul, Y. H. Kerr, A. Hahne, and S. Mecklenburg (2010), SMOS: The Challenging Sea Surface Salinity Measurement From Space, Proceedings of the IEEE, 98(5), 649-665.

Fore, A. G., S. H. Yueh, W. Tang, B. W. Stiles, and A. K. Hayashi (2016a), Combined active/passive retrievals of ocean vector wind and sea surface salinity with SMAP. IEEE Trans. Geosci. Remote Sens., 54(12):7396-7404, December 2016.

Fore, A., S. Yueh, W. Tang, and A. Hayashi, (2016b), SMAP Salinity and Wind Speed Data User's Guide Version 3.0, JPL, 30 December 2016.

Fournier, S., J. T. Reager, T. Lee, J. Vazquez-Cuervo, C. H. David, and M. M. Gierach (2016), SMAP observes flooding from land to sea: The Texas event of 2015, Geophys. Res. Lett., 43, 10,338-10,346, doi:10.1002/2016GL070821.

Fournier, S., Vialard, J., Lengaigne, M., Lee, T., Gierach, M. M., \& Chaitanya, A. V. S. (2017), Modulation of the Ganges-Brahmaputra river plume by the Indian Ocean dipole and eddies inferred from satellite observations. Journal of Geophysical Research: Oceans, 122, 9591-9604. https://doi.org/10.1002/2017JC013333.

Gaillard F. (2015), ISAS-13 temperature and salinity gridded fields. SEANOE. http://doi.org/10.17882/45945. 
991

992

993

994

995

996

997

998

999

1000

1001

1002

1003

1004

1005

1006

1007

1008

1009

1010

1011

1012

1013

1014

1015

1016

1017

1018

1019

1020

1021

1022

1023

1024

1025

1026

1027

1028

1029

1030

1031

Gaillard, F., T. Reynaud, V. Thierry, N. Kolodziejczyk and K. von Schuckmann (2016), In situ-Based Reanalysis of the Global Ocean Temperature and Salinity with ISAS: Variability of the Heat Content and Steric Height, J. Clim., 29, 1305-1323, doi:10.1175/JCLI-D-150028.1.

Grodsky, S. A., N. Reul, B. Chapron, J. A. Carton, and F. O. Bryan (2017), Interannual surface salinity on Northwest Atlantic shelf, J. Geophys. Res. Oceans, 122, 3638-3659, doi:10.1002/2016JC012580.

Hasson, A., T. Delcroix, J. Boutin, R. Dussin, and J. Ballabrera-Poy (2014), Analyzing the 2010-2011 La Niña signature in the tropical Pacific sea surface salinity using in situ data, SMOS observations, and a numerical simulation, Journal of Geophysical Research: Oceans, 119(6), 3855-3867, doi:10.1002/2013JC009388.

Hasson, A., M. Puy, J. Boutin, E. Guilyardi (2018), Northward Propagation across the Tropical North Pacific Ocean Revealed by Surface Salinity: How El Nino Anomalies Reach Hawaii?, Journal of Geophys. Res., in revision.

Hernandez, O., Boutin, J., Kolodziejczyk, N., Reverdin, G., Martin, N., Gaillard, F., Reul, N., \& Vergely, J.L. (2014). SMOS salinity in the subtropical North Atlantic salinity maximum: 1. Comparison with Aquarius and in situ salinity. Journal of Geophysical Research: Oceans, 119, 8878-8896.

Kerr, Y. H., et al. (2010), The SMOS Mission: New Tool for Monitoring Key Elements of the Global Water Cycle, Proceedings of the IEEE, 98(5), 666-687.

Kolodziejczyk, N., O. Hernandez, J. Boutin, and G. Reverdin (2015a), SMOS salinity in the subtropical North Atlantic salinity maximum: 2. Two-dimensional horizontal thermohaline variability, J. Geophys. Res. Oceans, 120, 972-987, doi:10.1002/2014JC010103.

Kolodziejczyk, N., G. Reverdin, J. Boutin, and O. Hernandez (2015b), Observation of the surface horizontal thermohaline variability at mesoscale to submesoscale in the north-eastern subtropical Atlantic Ocean, Journal of Geophysical Research: Oceans, 120(4), 2588-2600, doi:10.1002/2014JC010455.

Kolodziejczyk, N., J. Boutin, J.L. Vergely, S. Marchand, N. Martin, and G. Reverdin (2016). Mitigation of systematic errors in SMOS sea surface salinity. Remote Sensing of Environment, 180, 164-177.

Lagerloef, G., et al. (2008), The Aquarius/SAC-D mission: Designed to meet the salinity remote sensing challenge Oceanography, 21(1), 68-81.

Lagerloef, G. (2012), Satellite mission monitors ocean surface salinity, Eos Transactions of the AGU, 93(25), 233-234.

Meissner, T. and F. J. Wentz (2016), Remote Sensing Systems SMAP Ocean Surface Salinities [Level 2C, Level 3 Running 8-day, Level 3 Monthly], Version 2.0 validated release. Remote Sensing Systems, Santa Rosa, CA, USA. Available online at www.remss.com/missions/smap, doi: 10.5067/SMP20-3SPCS.

Mohammed, P. N. , M. Aksoy J. R. Piepmeier J. T. Johnson A. Bringer (2016), SMAP Lband microwave radiometer: RFI mitigation prelaunch analysis and first year on-orbit observations, IEEE Trans. Geosci. Remote Sens., vol. 54 no. 10 pp. 6035-6047. 
1032 Oliva, R., Daganzo-Eusebio, E., Kerr, Y., Mecklenburg, S., Nieto, S., Richaume, P., Gruhier, 1033 C. (2012), SMOS Radio frequency interference scenario: status and actions taken to improve the RFI environment in the 1400-1427-MHz passive band. IEEE Trans. Geosci. Remote Sens. 50 (5), 1427-1440.

1036 Olmedo, E., Martínez, J., Turiel, A., Ballabrera-Poy, J., \& Portabella, M. (2017), Debiased non-Bayesian retrieval: A novel approach to SMOS Sea Surface Salinity. Remote Sensing of Environment, 193, 103-126.

1039 Picaut, J., M. Ioualalen, T. Delcroix, F. Masia, R. Murtugudde, and J. Vialard (2001), The 1040 oceanic zone of convergence on the eastern edge of the Pacific warm pool: A synthesis of results and implications for ENSO and biogeochemical phenomena, J. Geophys. Res., 106, 2363-2386.

1043 Piepmeier J. R. et al., (2017), SMAP L-Band Microwave Radiometer: Instrument Design and 1044 First Year on Orbit," in IEEE Transactions on Geoscience and Remote Sensing, vol. 55, no. 4, pp. 1954-1966, doi: 10.1109/TGRS.2016.2631978.

1046 Reul N., and Coauthors (2014a), Sea Surface Salinity Observations from Space with the 1047 SMOS Satellite: A New Means to Monitor the Marine Branch of the Water Cycle. Surveys In 1048 Geophysics, 35(3), 681-722.

1049 Reul, N., B. Chapron, T. Lee, C. Donlon, J. Boutin, and G. Alory (2014b), Sea surface 1050 salinity structure of the meandering Gulf Stream revealed by SMOS sensor, Geophysical 1051

1052 Shenoi, S. S. C., D. Shankar (2002), Differences in heat budgets of the near-surface Arabian 1053 Sea and Bay of Bengal: Implications for the summer monsoon, J. Geophys. Res., 107(C6), 1054 doi:10.1029/2000JC000679,.

1055

1056

1057

1058

1059

SMOS-Ocean Expert Support Laboratories (2014), SMOS L2 OS Algorithm Theoretical Baseline Document, SO-TN-ARG-GS-0007, available on https://smos.argans.co.uk/docs/deliverables/delivered/ATBD/SO-TN-ARG-GS-0007_L2OSATBD_v3.11_140905.pdf.

Sommer, A., Reverdin, G., Kolodziejczyk, N., and Boutin, J. (2015), Sea Surface Salinity and Temperature Budgets in the North Atlantic Subtropical Gyre during SPURS Experiment: August 2012-August 2013. Frontiers in Marine Science, 2, 107.

1062 Spurgeon and SMOS-Ocean Expert Support Laboratories (2017), L2OS v662 Reprocessing 1063 Report, SO-RP-ARG-GS-0109, available on 1064 https://earth.esa.int/documents/10174/477987/SMOS-Level-2-Ocean-Salinity-v6621065 Reprocessing-Report/3587ea20-45d1-49a5-a6ca-084d1bf988b6?version=1.4.

1066 Supply, A., J. Boutin, J.-L. Vergely, N. Martin, A. Hasson, G. Reverdin, C. Mallet, N. Viltard 1067 1068 (2017), Precipitation Estimates from SMOS Sea Surface Salinity, QJRMS, doi:10.1002/qj.3110. 
Tang, W., A. Fore, S. Yueh, T. Lee, A. Hayashi, A. Sanchez-Franks, B. King, D. Baranowski, and J. Martinez (2017), Validating SMAP SSS with in-situ measurements. Remote Sensing Environ.. https://doi.org/10.1016/j.rse.2017.08.021.

Vergely and Boutin (2017), SMOS OS level 3: the Algorithm Theoretical Basis Document (v300), 05/05/2017, available on http://www.catds.fr/content/download/78841/1005020/file/ATBD_L3OS_v3.0.pdf?version=3

Vialard, J. and P. Delecluse (1998), An OGCM Study for the TOGA Decade. Part I : Role of Salinity in the Physics of the Western Pacific Fresh Pool. Journal of Physical Oceanography, 28, 1071 - 1088.

Yin, X., Boutin, J., \& Spurgeon, P. (2012). First assessment of SMOS data over open ocean: Part I; Pacific Ocean. IEEE Transactions on Geoscience and Remote Sensing, 50(5), 16481661.

Yin, X., J. Boutin, P. Spurgeon, Biases Between Measured and Simulated SMOS Brightness Temperatures Over Ocean: Influence of Sun (2013), IEEE Journal of Selected Topics in Applied Earth Observations and Remote Sensing, doi: 10.1109/JSTARS.2013.2252602.

Zine, S., J. Boutin, J. Font, N. Reul, P. Waldteufel, C. Gabarró, J. Tenerelli, F. Petitcolin, J. L. Vergely, M. Talone, and S. Delwart (2008), Overview of the SMOS sea surface salinity prototype processor, IEEE T. Geosci. Remote Sensing, 46, 621-645.

\section{List of Figure Captions:}

Figure 1 : Satellite SSS: SMOS SSS corrected according to (a, d, g, j) K2016 methodology, (b, e, h, k) the method described in this paper (CEC); (c, f, i, l) SMAP SSS. 4 case study areas : (a, b, c) : Bay of Bengal - August 21 2015 ; (d, e, f) : Gulf of Mexico - August18 2015 ; (g, h, i) : Eastern Tropical Atlantic Freshwater Pools - April 14 ${ }^{\text {th }} 2016$; (j, k, l) : Amazon plume - October 21 $1^{\text {st }} 2015$. SMOS and SMAP SSS are averaged over respectively a SMOS repetitive orbit sub-cycle (18 days) and two SMAP repetitive orbit cycles (16 days). Striking fresh SSS features in better agreement with SMOS (new version) and SMAP are indicated with black arrows.

Figure 2: Two examples of 2011-2016 latitudinal profiles of mean SSS (a; b) and of the standard deviation of the 2011-2016 monthly differences between SMOS SSS and ISAS SSS (c; d). The latitudinal means and standard deviations are computed over the Pacific Ocean 
1100 further than 1200 km from any coast: green: ISAS, blue: SMOS ascending orbits; red: SMOS

1101 descending orbits: a;c) November; middle of the swath (0-50 km from the center of the

1102 swath); b; d) January; edge of the swath (350-400 km from the center of the swath). Dashed

1103 vertical lines indicate $47^{\circ} \mathrm{N}$ and $47^{\circ} \mathrm{S}$.

1104 Figure 3: a) SSS variability ( $\left.\sigma_{\text {ssSnat }}\right)$ derived from 7 years of SMOS filtered and corrected

1105 SSS (after debiasing and filtering): large values are observed in river plumes and in rainy

1106 areas (ITCZ, SPCZ. b) Minimum and c) maximum of the SSS as derived from 18-day CEC

1107 LOCEAN that are used in the mapping of debiased near-real time products (see section 3.4).

1108 Figure 4: Monthly SMOS SSS compared to monthly ISAS SSS from July 2010 to December 2016.

1109 Standard deviation of the differences for a) L3P SMOS SSS; b) L3Q SMOS SSS. c) Number of

1110 months with differences between L3P and L3Q SMOS SSS greater than 0.2pss. d) Frequency with

1111 which corrections identified on Figure c) correspond to decreased bias with respect to ISAS (i.e. L3Q

1112 SMOS SSS closer to ISAS SSS than L3P SMOS SSS): red color means that the correction improves

1113 most of the time; blue color means that the correction degrades most of the time. Blank colors in

1114 figures c) and d) mean no change above the 0.2 pss threshold or no data in the L3P version (the

1115 comparison is done only for valid L3P SSS).

1116 Figure 5: Comparison of SMOS and SMAP 'weekly’ SSS: (a, d, g) r², (b, e, h) standard

1117 deviation of the differences, (c, f, i) number of pixels used in the comparisons. (a, b, c) 10-

1118 day L3P SMOS SSS, (d, e, f) 10-day L3Q SMOS SSS, (g, h, i) 9-day CEC SMOS SSS.

1119 Same indicators but when considering only the pixels available in the four products are

1120 presented in Appendix A2.

1121 Figure 6: Scatter plots of SMOS corrected fields versus SMAP SSS on the 4 regions and

1122 fresh events periods illustrated on Figure 1: first line: Bay of Bengal; $2^{\text {nd }}$ line: Gulf of

1123 Mexico; 3rd line : Eastern Tropical Atlantic Freshwater Pools; 4th line : Amazon plume. First 
1124 column: SMOS K2016 SSS; second column: SMOS 18-day CEC SSS; last column: SMOS 9day CEC SSS.

1126 Figure 7: Time series of statistical parameters computed over the Bay of Bengal case study 1127 area, April 2015 to December 2016: a) mean SSS; b) SSS standard deviation; c) square of the 1128 Pearson correlation coefficient $\left(\mathrm{r}^{2}\right)$ between SMOS and SMAP SSS; d) Standard deviation of 1129 the SMOS minus SMAP SSS differences (plain line) using L1 norm (dotted line). 'Weekly' 1130 SMOS CEC(blue), ‘bi-weekly’ SMOS CEC (green), ‘bi-weekly’ SMOS K2016 (red), 1131 'weekly' SMAP (black).

1132 Figure 8: Time series of statistical parameters computed over the Gulf of Mexico case study area, 1133 April 2015 to December 2016: a) mean SSS; b) SSS standard deviation; c) square of the Pearson 1134 correlation coefficient (r2) between SMOS and SMAP SSS; d) Standard deviation of the SMOS 1135 minus SMAP SSS differences (plain line) using L1 norm (dotted line). 'Weekly' SMOS CEC(blue), ‘bi-weekly’ SMOS CEC (green), 'bi-weekly’ SMOS K2016 (red), ‘weekly’ SMAP (black).

1137 Figure 9: Time series of statistical parameters computed over the Eastern Tropical Atlantic Freshwater 1138 Pools case study area, April 2015 to December 2016: a) mean SSS; b) SSS standard deviation; c) 1139 square of the Pearson correlation coefficient (r2) between SMOS and SMAP SSS; d) Standard 1140 deviation of the SMOS minus SMAP SSS differences (plain line) using L1 norm (dotted line).

1141 'Weekly' SMOS CEC(blue), 'bi-weekly' SMOS CEC (green), ‘bi-weekly’ SMOS K2016 (red), 1142 'weekly' SMAP (black).

1143 Figure 10: Time series of statistical parameters over the Amazon plume case study area, April 2015 to

1144 December 2016: a) mean SSS; b) SSS standard deviation; c) square of the Pearson correlation 1145 coefficient (r2) between SMOS and SMAP SSS; d) Standard deviation of the SMOS minus SMAP 1146 SSS differences (plain line) using L1 norm (dotted line). 'Weekly' SMOS CEC(blue), 'bi-weekly’ 1147 SMOS CEC (green), ‘bi-weekly’ SMOS K2016 (red), ‘weekly’ SMAP (black). 
1148 Figure 11: Top: Density spectra; Bottom: Coherence between ship SSS and SMOS or ISAS SSS. The

1149 spatial frequency (1/wavelength $(\mathrm{km}))$ is indicated below the bottom plot, whereas the corresponding

1150 wavelengths $(\mathrm{km})$ are indicated above the top plot. Vertical dashed lines correspond to spatial

1151 frequencies regularly spaced in logarithmic coordinates. Northern subtropical Atlantic (see box on the

1152 color map) in 2013. Ship SSS measured on regular merchant ships transects (14 regular transects in

1153 2013) (black), ISAS SSS (green), 10-day SMOS L3P (blue line), 10-day SMOS L3Q (dashed blue

1154 line), 18-day SMOS CEC (red line), 9-day SMOS CEC (red dashed line).

1155 Figure 12: Statistics of ship comparisons (May 2010-August 2016) binned as a function of

1156 the distance from the nearest coast: top) mean difference; middle) standard deviation of the

1157 differences; the black line indicates the standard deviation of ship SSS in each class; bottom)

1158 number of pixels used in the comparisons. Left: considering only the SMOS pixels common

1159 to all versions; right: considering all pixels available in each version. Ship and SMOS SSS

1160 are integrated over $100 \mathrm{~km}$. Orange: monthly SMOS L3P ; pink : monthly SMOS L3Q; light

1161 blue : 18-day SMOS CEC; green : ISAS.

1162 Figure 13: Examples of comparisons between ship SSS (black stars line) and SMOS SSS: orange: non

1163 corrected (L3P), purple: monthly L3Q corrected, light blue :18-day CEC corrected; green : ISAS.

1164 Left) from 2014-08-21 to 2014-09-03, Matisse ship. Right) from 2013-08-21 to 2013-09-03, Santa

1165 Cruz ship. All SSS products have been smoothed over +/-50 km.

1166 Figure 14: Standard deviation of 'weekly' satellite SSS minus ISAS SSS between $47^{\circ} \mathrm{N}$ and

$116747^{\circ} \mathrm{S}$, over the year 2016. a) SMOS CEC, b) SMAP CAP.

1168 Figure 15: SSS latitudinal profiles in December 2011(top left), 2012 (top right), 2013 (bottom

1169 left), 2014 (bottom right) in the Atlantic Ocean (1200 km from continents)- SMOS ascending

1170 orbits (blue), descending orbits (red), ISAS(green). 
1171 Figure 16: SSS latitudinal profiles in December 2011(top left), 2012 (top right), 2013 (bottom

1172 left), 2014 (bottom right) in the Pacific Ocean (1200 km from continents)- SMOS ascending

1173 orbits (blue), descending orbits (red), ISAS(green).

1174 Figure 17: Median of SMOS minus ISAS SSS absolute differences as a function of dwell line 1175 location and year, for the month of January (left), May (middle) and September (right), for 1176 ascending (top) and descending (bottom) orbits. The black lines indicate the range of selected $1177 \mathrm{X}_{\text {swath. }}$

1178 Figure 18: 'Weekly' comparison of SMOS and SMAP SSS: (a, c, e) square of the Pearson 1179 correlation coefficient $\left(\mathrm{r}^{2}\right),(\mathrm{b}, \mathrm{d}, \mathrm{f})$ standard deviation of the difference. $(\mathrm{a}, \mathrm{b}) \mathrm{L}$ ) SMOS 1180 SSS, (c, d) L3Q SMOS SSS, (e, f) CEC SMOS SSS. Only pixels common to the four 1181 products are considered in the comparisons.

Appendix A1: Selection of the region and of the reference $X_{\text {swath }}$ to be used for

1186 Given the high RFI contamination in the northern latitudes of the Atlantic Ocean and given

1187 the relatively small area further than $1000 \mathrm{~km}$ from the continents in the Atlantic Ocean, we

1188 choose to estimate the seasonally-varying latitudinal biases from Pacific Ocean orbits only.

1189 Nevertheless, before doing this choice, we checked, on $\mathrm{x}_{\text {swath }}$ and periods not very affected by

1190 RFI at high latitudes, that biases are similar in the Pacific and Atlantic Ocean. We observe

1191 that the differences between ocean basins are on the same order of magnitude as the

1192 interannual variability of the biases as illustrated with a few examples on Figure 15 and on 1193 Figure 16. 

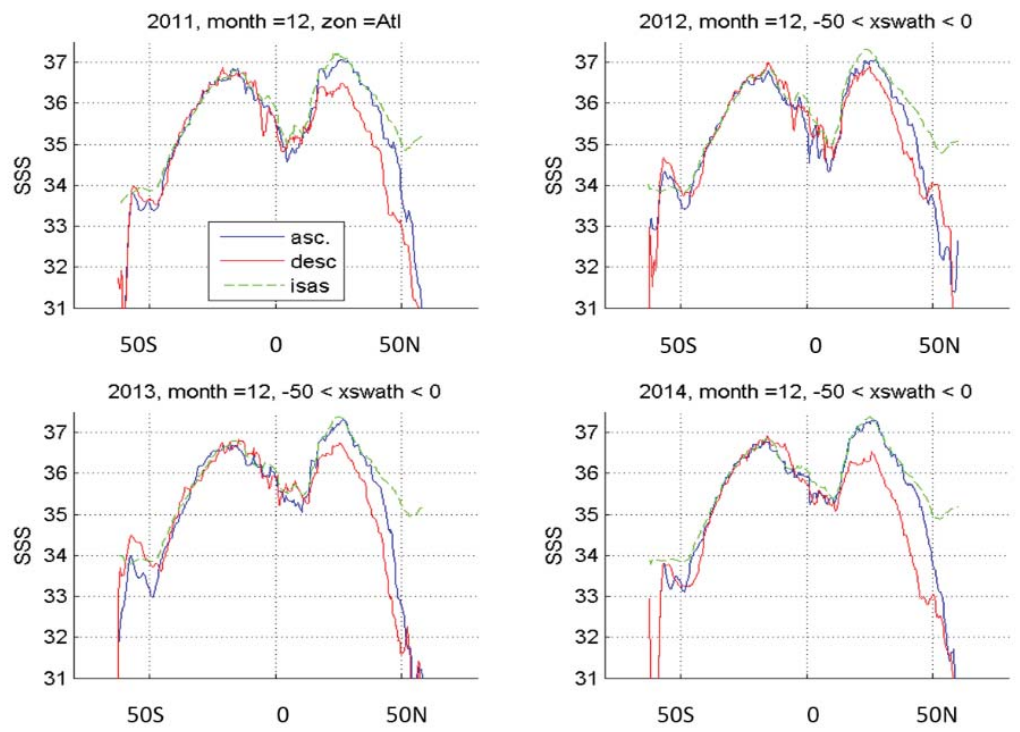

Figure 15: SSS latitudinal profiles in December 2011(top left), 2012 (top right), 2013 (bottom left), 2014 (bottom right) in the Atlantic Ocean (1200 km from continents)- SMOS ascending orbits (blue), descending orbits (red), ISAS(green).
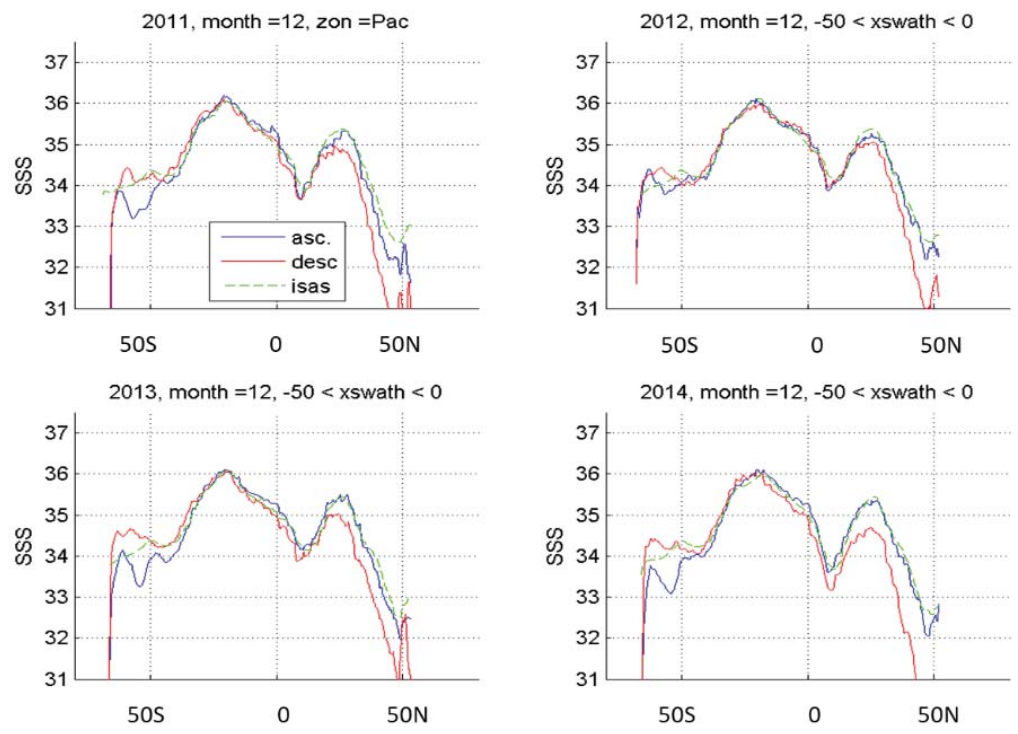

Figure 16: SSS latitudinal profiles in December 2011(top left), 2012 (top right), 2013 (bottom left), 2014 (bottom right) in the Pacific Ocean (1200 km from continents)- SMOS ascending orbits (blue), descending orbits (red), ISAS(green).

1204 Over the 2011-2016 period, for each $\mathrm{x}_{\text {swath, }}$ each month and each $\mathrm{x}_{\text {orb }}$, reference $\mathrm{X}_{\text {swath }}$ are

1205 chosen as the ones having relatively weak and stable (from one year to another) SMOS minus

1206 ISAS SSS differences (DIFF) over the $45^{\circ} \mathrm{S}-45^{\circ} \mathrm{N}$ latitudinal range. We did not define a 
quantitative criterion for this selection because the patterns of DIFF strongly vary from one

1208 month to another, from ascending to descending orbits and as a function of latitude (not

1209 shown). During most months, reference $\mathrm{x}_{\text {swath }}$ are located on ascending orbits only. We

1210 illustrate the location of the reference $\mathrm{x}_{\text {swath }}$ with respect to the median of SMOS minus ISAS

1211 SSS absolute differences for the months of January, May and September (Figure 17). The

1212 locations of all the selected reference $\mathrm{x}_{\text {swath }}$ are reported in Table 4.
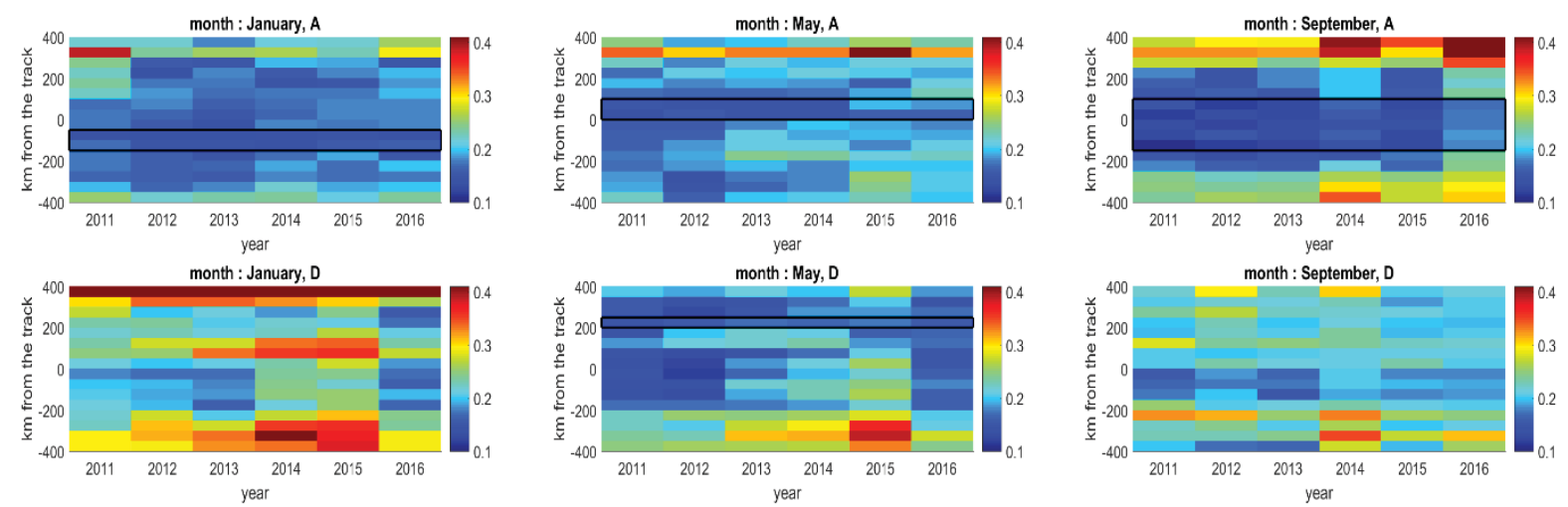

1213 Figure 17: Median of SMOS minus ISAS SSS absolute differences as a function of dwell line location 1214 and year, for the month of January (left), May (middle) and September (right), for ascending (top) and 1215 descending (bottom) orbits. The black lines indicate the range of selected $\mathrm{x}_{\text {swath. }}$.

1217 Table 4: Reference $\mathrm{x}_{\text {swath }}$ locations

\begin{tabular}{|c|c|c|}
\hline & Ascending orbits & Descending orbits \\
\hline January & {$[-150-50] \mathrm{km}$} & - \\
\hline February & {$[-250-100] \mathrm{km}$} & - \\
\hline March & {$[-250-100] \mathrm{km}$} & - \\
\hline April & {$\left[\begin{array}{ll}0 & 100\end{array}\right] \mathrm{km}$} & [150 200] km \\
\hline May & {$\left[\begin{array}{ll}0 & 100] \mathrm{km}\end{array}\right.$} & [200 250] km \\
\hline June & {$\left[\begin{array}{ll}50 & 100] \\
\mathrm{km}\end{array}\right.$} & {$\left[\begin{array}{ll}50 & 100] \mathrm{km}\end{array}\right.$} \\
\hline
\end{tabular}




\begin{tabular}{|c|c|c|}
\hline July & {$[-150$ 50] km } & {$\left[\begin{array}{lll}50 & 100\end{array}\right] \mathrm{km}$} \\
\hline August & {$\left[\begin{array}{lll}-250 & 250] \\
\mathrm{km}\end{array}\right.$} & {$\left[\begin{array}{lll}-50 & 100\end{array} \mathrm{~km}\right.$} \\
\hline September & {$\left[\begin{array}{lll}-150 & 100] \\
\mathrm{km}\end{array}\right.$} & - \\
\hline October & {$\left[\begin{array}{lll}-50 & 100] \mathrm{km}\end{array}\right.$} & - \\
\hline November & {$[-250-100] \mathrm{km}$} & - \\
\hline December & {$[-100-50] \mathrm{km}$} & - \\
\hline
\end{tabular}

1218

1219

1220 Appendix A2: SMOS-SMAP SSS comparison considering only pixels common

1221 to all SSS fields:
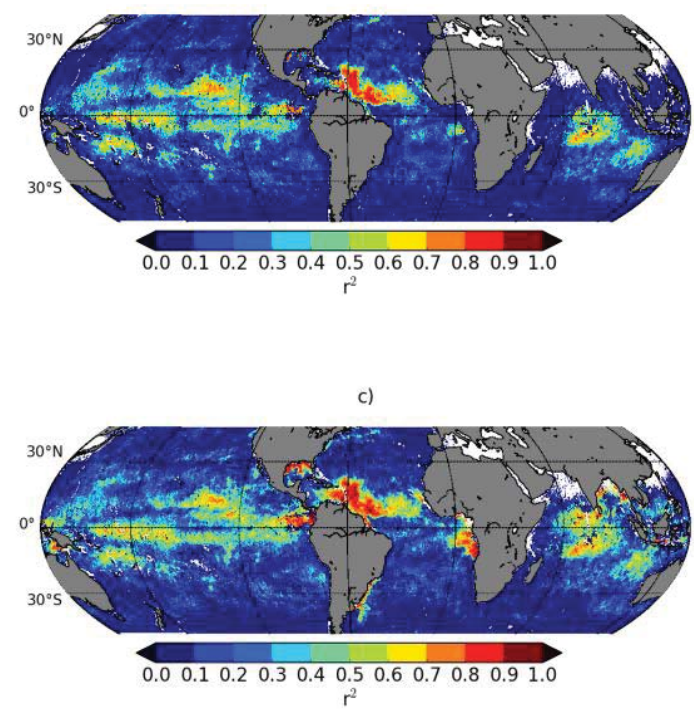

e)

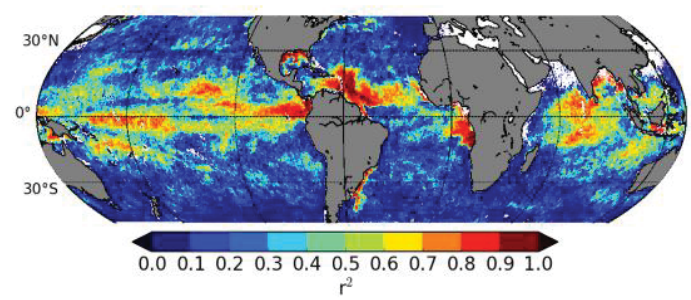

b)

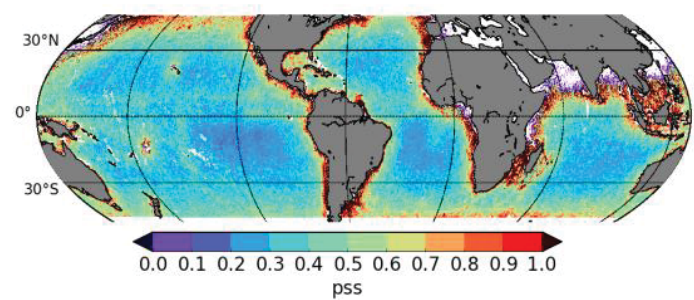

d)

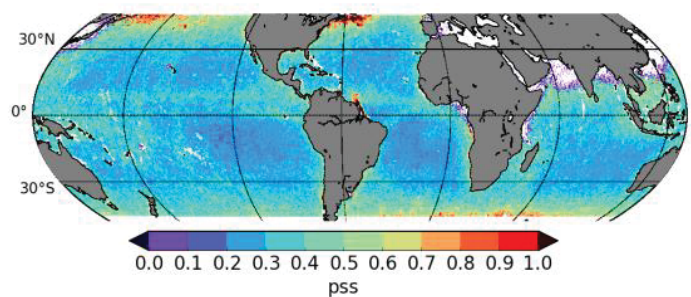

f)

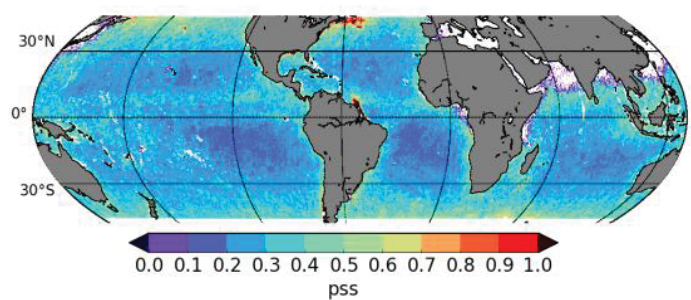

pss 
1222 Figure 18: 'Weekly' comparison of SMOS and SMAP SSS: (a, c, e) square of the Pearson 1223 correlation coefficient $\left(\mathrm{r}^{2}\right)$, (b, d, f) standard deviation of the difference. (a, b) L3P SMOS 1224 SSS, (c, d) L3Q SMOS SSS, (e, f) CEC SMOS SSS. Only pixels common to the four 1225 products are considered in the comparisons. 